\title{
Passivation, conductivity, and selectivity in solar cell contacts: concepts and simulations based on a unified partial-resistances framework
}

\author{
Arthur Onno, ${ }^{1}$ Christopher Chen, ${ }^{1}$ Priyaranga Koswatta, ${ }^{2, a)}$ Mathieu Boccard, ${ }^{3, a)}$ \\ and Zachary C. Holman ${ }^{1, b)}$ \\ ${ }^{1}$ School of Electrical, Computer and Energy Engineering, Arizona State University, Tempe, Arizona, 85287, USA \\ ${ }^{2}$ Intel Corporation, 3585 SW $198^{\text {th }}$ Avenue, Aloha, Oregon, 97007, USA \\ ${ }^{3}$ Photovoltaics and Thin-Film Electronics Laboratory (PV-Lab), Institute of Microengineering (IMT), Ecole \\ Polytechnique Fédérale de Lausanne (EPFL), Neuchâtel 2002, Switzerland
}

Passivation, conductivity, and selectivity are often acknowledged as the three requirements for optimal contacts to photovoltaic solar cells. Although there are generally accepted definitions and metrics for passivation and conductivity, a common understanding of the concept of selectivity is only now emerging. In this contribution, we present a generalized model of solar cell contacts based on the distinct lumped resistances encountered by electrons and holes traversing a contact, which we refer to as partial specific contact resistances. The relations between electron and hole partial current densities, quasi-Fermi level separation, and external voltage are derived from these partial specific contact resistances, leading to simple metrics for the aforementioned contact properties: the sum of the electron and hole resistances is a metric for passivation, their ratio a metric for selectivity, and the majority-carrier resistance a metric for conductivity. Using PC1D, we validate our model by simulating 10,500 cases of homojunction contacts to crystalline silicon solar cells, although our framework is material agnostic and can be equally applied to any other type of absorber. In these simulations, the hole contact and absorber are assumed to be ideal, whereas we vary the partial specific contact resistances in the electron contact by orders of magnitude by adjusting the electron and hole mobilities, their densities (through variations of the donor doping density), and the contact thickness. The simulations confirm the finding of the model that, when the contact fraction cannot be adjusted-as is the case with full-area contactscombined passivation and conductivity are necessary and sufficient for optimal solar cell performance, and they imply selectivity. However, the reciprocal is not true: contacts can be selective but lack conductivity—causing a deleterious drop in fill factor — or selective but provide poor passivation—-leading to a reduction in implied open-circuit voltage and, hence, actual open-circuit voltage. Thus, selectivity is a meaningful metric in the sole case of partial-area contacts, where the contact fraction can be adjusted arbitrarily.

\section{INTRODUCTION}

Photovoltaic solar cells are devices that convert thermal radiation into electric power, in the form of a current of charge carriers that have a usable free energy corresponding to the difference in electrical potential between the two electrodes of the

a) This research was performed while P. Koswatta and M. Boccard were at Arizona State University, Tempe, Arizona, 85287, USA.

b) Author to whom correspondence should be addressed. Electronic mail: zachary.holman@asu.edu. 
cell. This process is achieved in two steps: first, in the absorber, the thermal radiation is absorbed and converted into populations of electrons and holes with distinct electrochemical potentials, or quasi-Fermi levels (qFLs); then, in the contacts, these electrons and holes are selectively transported to their respective spatially separated electrodes. To ensure maximal power conversion, both of these processes must be carried out with maximal efficiency. ${ }^{1}$

The former process - generating and maintaining two separate populations of charge carriers through absorption and minimal non-radiative recombination — is understood to be quantified by the external radiative efficiency and to require excellent material quality and surface passivation..$^{2-4}$ The latter process - the separation of charge carriers and their collection at different locations - is inherently tied to the transport behavior of electrons and holes within the device. Internal built-in electric fields have long been held responsible for this separation of charge carriers in solar cells, although this idea has since been shown to be erroneous. ${ }^{1,5,6}$ Using basic yet exact physics, Würfel et al. have demonstrated how, at the microscopic scale within a solar cell, the currents of electrons and holes are driven by their respective conductivities and qFL gradients. ${ }^{6}$ Selective transport is, thus, the result of an asymmetry in electron and hole conductivities, the p-n junction being just one particular embodiment of such an asymmetry. Asymmetries in either carrier density or mobility can be exploited to separate charge carriers and, in a hypothetical case where the asymmetry in mobility is high enough, Würfel et al. showed that selective transport can be achieved without any doping at all.

The photovoltaic community has come to an understanding that the contacts of a solar cell should drive this selective transport, and the associated carrier separation, by providing the asymmetries in electron and holes conductivities. As Glunz et al. and we have previously presented, ${ }^{7-10}$ such contacts, which are often called "carrier-selective", must be passivating, conductive, and selective, on top of being optically optimized (transparent for a front contact, transparent or reflective for a back contact). Each of these qualities is associated with maximizing an important cell parameter: passivation leads to a high qFL separation —and thus implied open-circuit voltage $\left(i V_{o c}\right)$ —within the absorber; conductivity ensures minimal resistive losses at the maximum power point and, hence, a high fill factor $(F F)$; and selectivity limits voltage losses within the contact, leading to an actual open-circuit voltage $\left(V_{o c}\right)$ approaching or even equaling $i V_{o c}$. (Throughout the paper, we interchangeably use the terms "qFL separation" and "implied voltage $(i V)$ ", as these two quantities are directly proportional through the elementary charge $q$ : qFL separation $=q \times i V$.) Agreed-upon measurable metrics exist for passivation and conductivity, in the form of the contact recombination current density $j_{0, c}{ }^{5,11,12}$ and the specific contact resistance $\rho_{c},{ }^{13}$ respectively. This framework is sometimes referred to as the "lumped-skin" model. ${ }^{14}$ In the crystalline silicon (c-Si) solar cell community, contacts are commonly classified according to this taxonomy and mapped onto $j_{0, c}-\rho_{c}$ contour plots, ${ }^{15}$ though the link between the local asymmetry in conductivities ${ }^{1,6}$ and the device-level $j_{0, c}$ and $\rho_{c}$ metrics is far from evident. 
Conversely, the debate is still open regarding selectivity, with multiple definitions, models, and metrics proposed. ${ }^{16-21}$ Brendel et al. ${ }^{16}$ define selectivity as the ability to block one type of carrier while efficiently conducting the other. They make use of the lumped-skin model parameters $j_{0, c}$ and $\rho_{c}$ to define their selectivity metric: $S=k_{B} T /\left(q j_{0, c} \rho_{c}\right)$, where $k_{B} T / q$ is the thermal voltage with $k_{B}$ the Boltzmann constant and $T$ the temperature. This definition is practical, as it relies on readily measurable metrics and it does not require separation of recombination at the absorber/contact interface (related to surface passivation), within the contact (related to contact material quality and bulk resistance), and at the contact/metallic electrode interface (related to the total resistance encountered by the carriers). Using this definition, Brendel et al. ${ }^{16}$ demonstrated that, provided one is able to choose the optimal contact fraction, solar cell efficiency is a function of the selectivity alone: two different contact schemes with equal selectivity may need different contact fractions to reach maximal efficiency but this maximal efficiency will be the same. This is also visible in Bullock's $j_{0, c}-\rho_{c}$ plots. ${ }^{15}$ In a similar yet distinct approach, Roe $e t$ al. ${ }^{17,18}$ use a "partial diodes" model, where both charge-carrier partial current densities behave according to diode equations, leading to four saturation current densities for a given device: the electron and hole saturation current densities for the left $\operatorname{contact}\left(j_{0, e}^{l}\right.$ and $\left.j_{0, h}^{l}\right)$ and for the right contact $\left(j_{0, e}^{r}\right.$, and $\left.j_{0, h}^{r}\right)$. The authors define two types of selectivities: (1) A contact selectivity, corresponding to the ratio between the majority- and minority-carrier partial saturation current densities $\left(S^{l}=\right.$ $j_{0, h}^{l} / j_{0, e}^{l}$ and $S^{r}=j_{0, e}^{r} / j_{0, h}^{r}$, assuming a left hole contact and a right electron contact); ${ }^{17}$ and (2) electron and hole device-level selectivities, which correspond to the ratio of the electron partial saturation current densities at each contact and the hole partial saturation current densities at each contact $\left(S_{e}=j_{0, e}^{r} / j_{0, e}^{l}\right.$ and $S_{h}=j_{0, h}^{l} / j_{0, h}^{r}$, assuming again a left hole contact and a right electron contact). ${ }^{18}$ The former describes the asymmetry between the kinetics of electron and holes at a given contact; the latter describes the asymmetry between kinetics at each contact for a given type of charge carrier. In particular, for contact-limited devices, the device-level selectivities determine the shape of the $J-V$ curve. That is, the performance of the cell is not dictated by a unique contact but by the interplay between the two contacts.

Although the approaches from Brendel et al. and Roe et al. have their merits, both include restrictive assumptions. A common limitation is that they rely on ideal diode behavior, with $j_{0}$ independent of voltage and an ideality factor of $m=1$, for at least one charge carrier. This ideal behavior is not always present in practice: determining constant $j_{0}$ values from measurements can be challenging, if not impossible, ${ }^{14,21}$ rendering the above definitions of selectivity problematic for devices with non-linear interfacial recombination processes. Additionally, the lumped-skin model used by Brendel et al. assumes the recombination and resistive processes to be independent (i.e. in series), although we will show that they are in fact interdependent. As a result, the model cannot account for voltage losses at open-circuit $\left(i V_{o c}>V_{o c}\right)$ or S-shaped $J-V$ curves, which are common with perovskite and CdTe devices as well as with novel hetero-contacts to c-Si solar cells. ${ }^{22,23}$ Furthermore, 
in that approach, the polarity of the contact is decided a-priori and, hence, the symmetry between electrons and holes—present in Würfel's generalized local conductivity approach ${ }^{1,6}$ —is lost. The model from Roe et al. can explain phenomena such as Sshaped curves and $i V_{o c}>V_{o c}$ quite well and conserves the symmetry between electrons and holes. However, it ignores finitemobility and resistance effects — the basis of carrier separation in Würfel's approach—and instead assumes constant qFLs in the absorber and a constant Fermi level in the contact, with a discontinuity across the interface (i.e. abrupt collapse of the qFL separation, with no gradients of the qFLs).

In this work, we propose a generalized model that is able to describe cases where the contact recombination rate is not a linear function of the carrier density. That is, it is applicable in instances when the contact saturation current density $j_{0, c}$ used by Brendel et al. or the partial saturation currents densities $j_{0, e}$ and $j_{0, h}$ used by Roe et al. are not constant and instead depend on the injection level. Consistent with the microscopic conductivity theory developed by Würfel et al., ${ }^{1,6}$ our model integrates the partial current-density equations over the thickness of the contact. Thus, we express the spatial variations in qFLs for both electrons and holes as a function of their partial current densities, and we define corresponding voltage-dependent partial specific contact resistances. These electron and hole resistances, in turn, are used to calculate $i V_{o c}$ and $V_{o c}$ of a solar cell having this contact, as well as their ratio $V_{o c} / i V_{o c}$. We adopt this ratio as a readily measurable metric of a contact's selectivity, which we understand to be its ability to minimize the voltage drop across it at open circuit, in agreement with Glunz et al. ${ }^{10}$ Reciprocally, the values of the partial specific contact resistances at open circuit can be calculated from $i V_{o c}$ and $V_{o c}$. We validate this model through simulation of $10,500 \mathrm{c}-\mathrm{Si}$ homojunction devices in PC1D, ${ }^{24,25}$ although our approach is general and not limited to a particular absorber material or device structure.

\section{CONCEPT AND THEORETICAL MODEL}

\section{A. Definitions and assumptions}

The term "contact" has often been used in the literature with varying meaning, but always involves a metal or transparent conductive oxide (TCO) electrode that connects the device to an external load. Indeed, every solar cell must have two of these, spatially separated, to collect electrons at one location and holes at the other. Since metals and TCOs do not support separate qFLs, their surfaces must facilitate recombination, and we define this recombination-active interface at which only one Fermi level is allowed as the outer edge of the contact. The inner edge of the contact is usually harder to define since it can engulf part of the absorber. In the ideal case of uniform generation and recombination rates throughout the absorber or of diffusion lengths considerably longer than the thickness of the absorber, the qFL separation will be spatially quasi-constant within the absorber and the necessary qFL collapse — from two distinct levels in the absorber to a unique level in the metallic electrodewill occur entirely across the contact. Thus, we define a contact as a region designed to facilitate this qFL collapse: Beyond the 
inner edge of the contact, the qFL separation is ideally quasi-constant and equal to $i V_{o c}$; at the outer edge of the contact, only one Fermi-level, corresponding to the potential of the positive or negative electrode of the solar cell, is allowed. In practice, for weakly passivating contact structures (e.g. aluminum back-surface fields to c-Si cells), a non-negligible fraction of the qFL collapse can happen in the absorber, as we will show in Section IV. However, the majority of modern cell designs incorporate one or more layers between the absorber and the electrode that is designed to support most of the qFL collapse, and thus these layers are appropriately called the "contacts".

Local or partial-area contacts, which cover — and, thus, extract current from—only part of the cell surface, are possible and even common with c-Si cells (e.g. PERC architectures). When they are used, areal contact fraction is then an important design parameter. ${ }^{16}$ However, local contacts increase manufacturing complexity and thus cost, and they are not practical for highsheet-resistance thin-film absorbers because contact openings cannot be spaced close enough together to avoid unacceptable series resistance. We thus restrict the following analysis to full-area contacts (unity contact fraction), mirroring the present search-across absorber technologies in the photovoltaics research community-for heterojunction "carrier-selective" contacts. This constraint has far-reaching implications on the design requirements for optimal contacts, as we will demonstrate and was previously reported by Brendel et al. ${ }^{16,26}$

In order to segregate intrinsic, contact-specific recombination and resistive processes from extrinsic sources of inefficiencies, we consider an ideal test structure in which radiative and Auger recombination dominate in the absorber and surface recombination is absent at the absorber/contact interfaces (i.e. excellent absorber material quality and surface passivation). Moreover, we neglect absorption and recombination within the contacts themselves and we consider the system in steady state. As a result, generation is confined to the absorber and recombination occurs only through intrinsic processes within the absorber or at the contact/electrode interfaces, where only one Fermi level is allowed. In particular, at open circuit, all photo-generated electrons and holes have to recombine within the absorber or at the metal interface. Finally, we limit our model to one dimension. This simplified analytical treatment does not invalidate the application of the theory to (more complex) practical cases: the framework we present can be easily extended to non-ideal devices suffering from defect-induced or surface recombination, and devices for which absorption or recombination within the contacts is non-negligible.

\section{B. Partial conductivities}

Throughout this study, we often consider "partial" metrics (e.g. partial current densities or partial resistances), meaning that they refer to the metric for a unique type of carrier: electrons or holes. We denote these partial metrics with subscript $e$ and $h$ to refer to electrons and holes, respectively. $j_{e}$ is, thus, the partial current density of electrons, whereas $j_{h}$ is the partial current 
density of holes. As pointed out by Würfel et al. in their explanation of charge carrier separation, each partial current density is equal to the product of the conductivity and the gradient of electrochemical potential: ${ }^{1,6}$

$$
\begin{aligned}
& j_{e}=\frac{\sigma_{e}}{q} \nabla E_{F, e}=\frac{\sigma_{e}}{q} \frac{d E_{F, e}}{d x}=\mu_{e} n_{e} \frac{d E_{F, e}}{d x}, \\
& j_{h}=\frac{\sigma_{h}}{q} \nabla E_{F, h}=\frac{\sigma_{h}}{q} \frac{d E_{F, h}}{d x}=\mu_{h} n_{h} \frac{d E_{F, h}}{d x},
\end{aligned}
$$

where $\sigma_{e / h}$ are the electron and hole partial conductivities, $E_{F, e / h}$ are their qFLs, and $q$ is the elementary charge. The partial conductivities are the product of the charge carrier mobilities $\mu_{e / h}$, their densities $n_{e / h}$, and the elementary charge:

$$
\begin{aligned}
& \sigma_{e}=q \mu_{e} n_{e}, \\
& \sigma_{h}=q \mu_{h} n_{h} .
\end{aligned}
$$

Note that the carrier mobilities and densities, and consequently the partial conductivities, can all vary spatially, as detailed in Appendix A. In particular, the partial conductivities are not constant throughout the contacts. However, the local conductivity relations expressed in Equation 1 are valid at all points in space for free electrons and holes in any material; thus, they can be applied to any absorber as well as to a wide range of contact structures, including heterostructures. As described by Würfel $e t$ $a l .{ }^{1,6}$ the sum of the partial currents must be null in a solar cell at open circuit, and thus a qFL gradient for one type of charge carrier must be counterbalanced by a qFL gradient for the other type, with a proportionality factor equal to the ratio of partial conductivities. That is, both types of charge carriers contribute to the qFL collapse, but an asymmetry in partial conductivities within the contact will make this collapse unevenly split between the electron and hole qFLs. This, in turn, will drive the selective extraction of one type of charge carrier at operating points away from open circuit. Charge carrier mobility and density are both levers to manipulate the partial conductivities, although, in practice, the mobility is difficult to adjust whereas the density can be tuned by modifying the contact's work function through extrinsic doping or modulation of intrinsic defects with shallow states. ${ }^{27}$ As a result, asymmetries in mobilities tend to be overlooked - in part because they cannot be displayed in band diagrams - although they contribute to the overall ratio of partial conductivities.

\section{Partial specific contact resistances}

Moving beyond Würfel et al.'s framework, we define partial specific contact resistances $\rho_{c, e / h}$ by integrating the local conductivity equations — which can equivalently be considered as local resistivity relations $\left(\rho_{e / h}=1 / \sigma_{e / h}\right)$ - over the width of a contact $L_{c}$. As shown in Appendix A, this is equivalent to expressing the electrochemical potential drop across the contact for electrons/holes $\Delta_{c} V_{e / h}$ as the product of their respective partial current densities $j_{e / h}$ through the contact and partial specific contact resistances $\rho_{c, e / h}$ : 


$$
\begin{aligned}
& \Delta_{\mathrm{c}} V_{e}=\frac{1}{q} \times\left(E_{F, e}-E_{F, m}\right)=\frac{L_{c}}{\overline{\sigma_{e}}} j_{e}=\rho_{c, e} j_{e}, \\
& \Delta_{c} V_{h}=\frac{1}{q} \times\left(E_{F, h}-E_{F, m}\right)=\frac{L_{c}}{\bar{\sigma}_{h}} j_{h}=\rho_{c, h} j_{h},
\end{aligned}
$$

with $E_{F, m}$ the sole Fermi level in the metallic electrode, $E_{F, e / h}$ the qFL of electrons/holes in the absorber, and $\overline{\sigma_{e, h}}$ the average conductivity as a function of the charge-carrier qFL:

$$
\begin{aligned}
& \overline{\sigma_{e}}=\frac{q}{E_{F, e}-E_{F, m}} \int_{\substack{E_{F}=E_{F, e} \\
E_{F}=E_{F, m}}}^{E_{F}=E_{F, m}} \mu_{e}\left(E_{F}\right) n_{e}\left(E_{F}\right) d E_{F}=\frac{1}{E_{F, e}-E_{F, m}} \int_{\substack{E_{F}=E_{F, e} \\
E_{F}=E_{F, m}}}^{E_{F}=E_{F, m}} \sigma_{e}\left(E_{F}\right) d E_{F}, \\
& \overline{\sigma_{h}}=\frac{q}{E_{F, h}-E_{F, m}} \int_{E_{F}=E_{F, h}}^{E_{F}=E_{F, m}} \mu_{h}\left(E_{F}\right) n_{h}\left(E_{F}\right) d E_{F}=\frac{1}{E_{F, h}-E_{F, m}} \int_{E_{F}=E_{F, h}}^{E_{F}=E_{F, m}} \sigma_{h}\left(E_{F}\right) d E_{F} .
\end{aligned}
$$

The partial specific contact resistances $\rho_{c, e / h}$ can be calculated from Equation (3) knowing the contact thickness $L_{c}$ and average conductivities $\overline{\sigma_{e / h}}$ or knowing the partial voltage drops $\Delta_{c} V_{e / h}$ and partial current densities $j_{e / h}$ :

$$
\begin{gathered}
\rho_{c, e}=\frac{\Delta_{c} V_{e}}{j_{e}}, \\
\rho_{c, h}=\frac{\Delta_{c} V_{h}}{j_{h}} .
\end{gathered}
$$

For ease of calculation, we chose the latter option in the simulations described in Section III. For cases where the mobility $\mu_{e / h}$ is constant in space - and, thus, with the $\mathrm{qFL} E_{F, e / h}$ — such as in Sections III and IV, that term can be extracted from the integral in Equation (4) and only the carrier density as a function of qFL needs integration.

Note that, though the specific contact resistance $\rho_{c, e / h}$ shares a symbol with the partial resistivity $\rho_{e / h}$, they have different meanings and units. The partial resistivity $\rho_{e / h}$ is a local physical quantity, measured in $\Omega . \mathrm{cm}$, and is isotropic in nonpathological cases. The partial specific contact resistance $\rho_{c, e / h}$, with the $c$ index, is a device-level physical quantity, measured in $\Omega . \mathrm{cm}^{2}$, and is tied to a restricted contact structure with well-defined surface boundaries and, in the planar case, a specific direction. Also, note that these partial specific contact resistances depend on the solar cell's operating point and are thus functions of the implied voltage: $\rho_{c, e / h}(i V)$. In particular, cases where these partial specific contact resistances behave as diodes have been described in a separate publication. ${ }^{28}$ For the sake of clarity, we have thus far limited our analysis to the ideal case where the qFL collapse is restricted to the contact region, where generation and recombination are assumed to be negligible. However, our approach can be extended to cases where partial voltage drops are present in the absorber by considering additional partial specific absorber resistances $\rho_{a b s, e / h}$, as is presented in Appendix A and used in Sections III and IV. Furthermore, although the analysis would appear to be inapplicable to contact structures relying on tunneling for majoritycarrier transport - e.g., through a thin silicon oxide layer, as in TOPCon solar cells ${ }^{29}$ - the treatment of such structures is also possible using our partial specific resistances framework, as detailed in Appendix A. Finally, the case of cells with non- 
negligible surface recombination at the absorber/contact interface can be treated by considering a voltage-dependent shunt resistance in parallel of the absorber, as described in the Supplementary Material (Figure S1).

This device-level specific contact resistance approach is summarized graphically in Figure 1, which overlays a traditional band diagram representation of a solar cell with an equivalent electrical circuit. The superscript indices $l$ and $r$ denote the left (hole) and right (electron) contacts, respectively. The absorber-related generation and recombination processes are represented by a current generator and a diode, respectively (multiple diodes may be used for multiple recombination processes). For both left and right contacts, the partial specific contact resistances are represented by red resistors, the lengths of which depend on the resistance value. Each recombination-active contact/electrode interface acts as a shunt of the absorber's generator-diode couple. As a result, the partial specific contact resistances "pry open" the qFLs to maintain the separation and, if both resistances of one contact are too low, the overall contact shunt resistance is low and $i V$ is reduced. It is important to note that the resistances are injection dependent and that, consequently, this shunt is non-ohmic. The external voltage is proportional to the difference of the Fermi levels in the left and right electrodes, $E_{F, m}^{r}-E_{F, m}^{l}$. These Fermi levels in the electrodes depend on $i V$ and on how the qFL collapse is distributed at each contact between a voltage drop for electrons and a voltage drop for holes: the resistances act as a voltage divider.

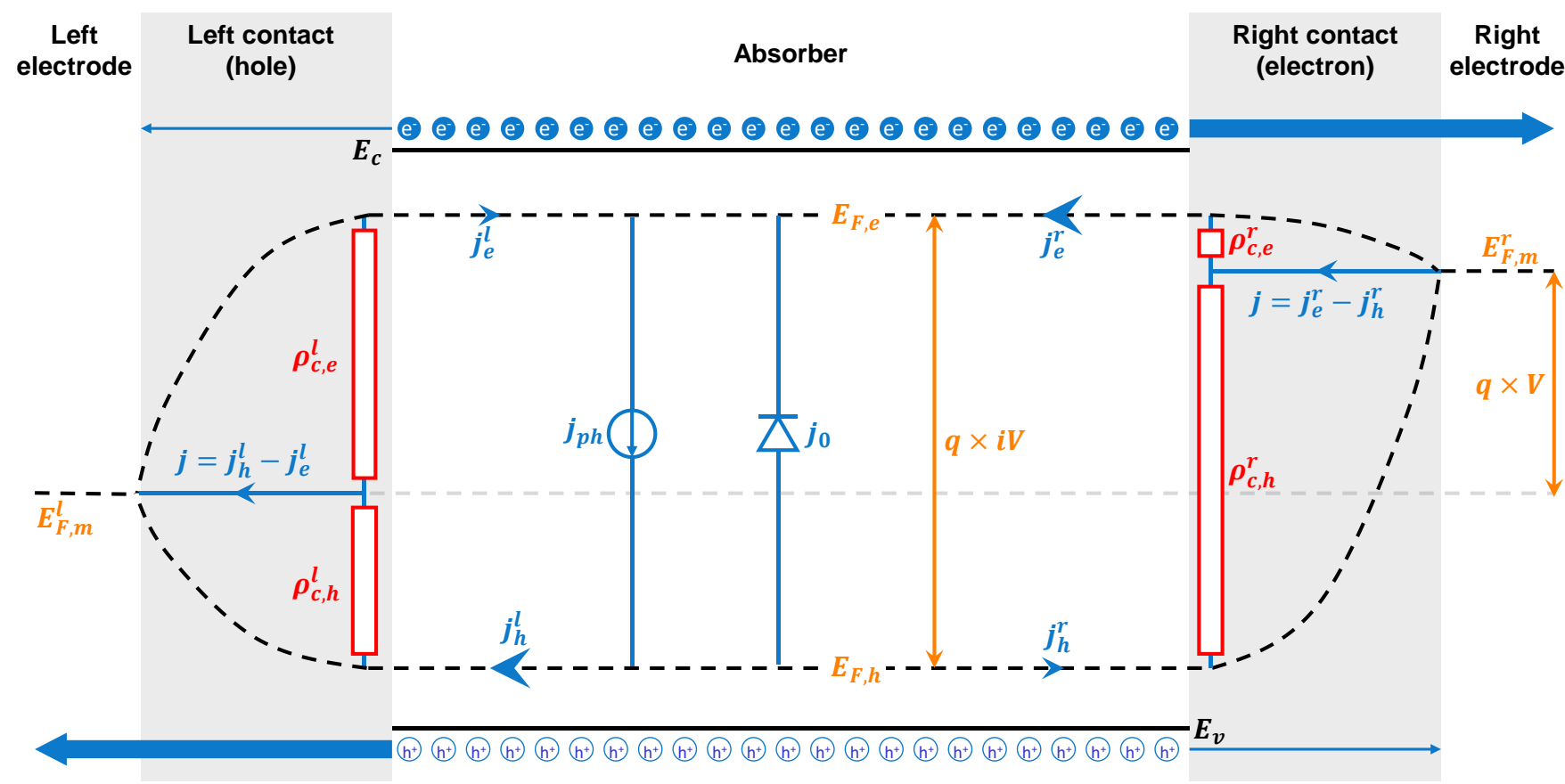

FIG. 1. Representation of the partial specific contact resistance model for an operating solar cell, overlaying a band diagram and an electrical circuit. The electrodes are displayed at the extreme left and right of the figure, separated from the central absorber by contacts (shaded gray). The qFL separation is quasi-constant in the absorber-due to the generation and recombination processes represented by a blue current generator and a blue diode, respectively - and it collapses in the contacts to the unique Fermi levels of the electrodes. "Minority carrier" recombination current densities $j_{h}^{r}$ and $j_{e}^{l}$, denoted with small blue arrows, flow through both contacts. "Majority carrier" current densities $j_{h}^{l}$ and $j_{e}^{r}$, denoted with large blue arrows, similarly flow to the electrodes through the contacts, ideally with a negligible reduction in qFL. The partial specific contact resistances are represented by red resistors. The length of each resistor is proportional to its resistance value. 


\section{Passivation}

At open circuit, the partial current densities of electrons and holes have to match in order to ensure a total current density equal to zero. Assuming an absorber with a photo-generated current density $j_{p h}$ and a linear combination of $p$ monomial recombination processes with associated dark recombination current densities $j_{0, k}$ and ideality factors $m_{k}$-these recombination processes being limited to radiative and Auger recombination in our ideal test case - the classic diode equation, taking into account our partial specific contact resistances, becomes:

$$
j=0=j_{p h}+\sum_{k=1}^{p} j_{0, k}\left(1-\exp \left(\frac{q \times i V_{o c}}{m_{k} k_{B} T}\right)\right)-\frac{i V_{o c}}{\rho_{c, e}^{l}+\rho_{c, h}^{l}}-\frac{i V_{o c}}{\rho_{c, e}^{r}+\rho_{c, h}^{r}} .
$$

Thus, the implied open-circuit voltage $i V_{o c}$ depends symmetrically on the sums of the partial specific contact resistances in each contact. Naturally, the sums are indifferent to the type of charge carrier: either contact can be an electron contact, a hole contact, or non-selective towards charge carriers without any impact on $i V_{o c}$ as long as the sum of the contact's partial specific contact resistances remains the same. We can therefore define a full-device passivation metric as:

$$
\rho_{c, t o t}=\frac{\left(\rho_{c, e}^{l}+\rho_{c, h}^{l}\right)\left(\rho_{c, e}^{r}+\rho_{c, h}^{r}\right)}{\rho_{c, e}^{l}+\rho_{c, h}^{l}+\rho_{c, e}^{r}+\rho_{c, h}^{r}} .
$$

This characteristic specific contact resistance is the equivalent total shunt resistance the absorber is subjected to. In the ideal case, it would be infinite. In practical cases, $\rho_{c, t o t}>1000 \times\left(m k_{B} T\right) /\left(q j_{p h}\right)$-with $m$ the ideality factor of the dominant recombination process within the absorber-ensures a negligible $(<0.1 \%)$ reduction of the achievable $i V_{o c}$, even when considering a perfect absorber with only radiative recombination. The full derivation of this approximation is detailed in Appendix B. For a hypothetical test structure with an ideal left hole contact $\left(\rho_{c, e}^{l}+\rho_{c, h}^{l}=\infty\right)$ and a non-ideal right electron contact $\left(0<\rho_{c, e}^{r}+\rho_{c, h}^{r}<\infty\right)$, such as the one simulated in Section IV, the relation simplifies to:

$$
\rho_{c, t o t}=\rho_{c, e}^{r}+\rho_{c, h}^{r}
$$

\section{E. Selectivity}

The relation between $i V_{o c}$ and $V_{o c}$ is straightforward, as the partial specific contact resistances act as voltage dividers:

$$
V_{o c}=\left[1-\frac{\rho_{c, h}^{l}}{\rho_{c, e}^{l}+\rho_{c, h}^{l}}-\frac{\rho_{c, e}^{r}}{\rho_{c, e}^{r}+\rho_{c, h}^{r}}\right] i V_{o c} .
$$

The sign of the term in square brackets indicates the direction of the current flow and the actual polarities of both electrodes. If positive, the current flows right to left: the left contact is a better hole contact than the right contact and the right contact is a better electron contact than the left contact, in agreement with Figure 1. Conversely, if negative, the current flows from left to 
right and the actual polarities of the contacts are swapped. For a given value of $i V_{o c}$, to achieve $V_{o c}$ as high as possible, the ratio of the partial specific contact resistances of electrons and holes $\rho_{c, e} / \rho_{c, h}$ must be high $(\gg 1)$ at one contact and low $(\ll 1)$ at the other. Again, in the case of a test structure with an ideal left hole contact and a non-ideal right electron contact, the relation simplifies to:

$$
V_{o c}=\left[1-\frac{\rho_{c, e}^{r}}{\rho_{c, e}^{r}+\rho_{c, h}^{r}}\right] i V_{o c}=\frac{\rho_{c, h}^{r}}{\rho_{c, e}^{r}+\rho_{c, h}^{r}} i V_{o c}=S_{e} \times i V_{o c} .
$$

Following our previous conception of selectivity ${ }^{7,9}$, shared by Glunz et al., ${ }^{10}$ as the ability to minimize the difference between $i V_{o c}$ and $V_{o c}$, the quantity $\rho_{c, h} /\left(\rho_{c, e}+\rho_{c, h}\right)$ is a metric of the electron selectivity $S_{e}$ of a contact and, symmetrically, $\rho_{c, e} /\left(\rho_{c, e}+\rho_{c, h}\right)$ is a metric of the hole selectivity $S_{h}$ of a contact. Note that our definition uses the sum of the resistances as the denominator whereas Brendel et al. use the smaller of the two resistances. As a consequence, our selectivity always takes values between 0 and 1 (and, for a given contact structure, $S_{e}+S_{h}=1$ ) whereas the selectivity from Brendel et al. can be any positive number up to infinity. ${ }^{16}$ In the ideal case, the selectivities are maximized so that $S_{h}=1$ for one contact- the hole contact-and $S_{e}=1$ for the other-the electron contact. Alternatively, it is also possible to invert this thinking and seek minimum selectivities towards "minority carriers" (carriers of the wrong type for a given contact): $S_{h}=\rho_{c, e} /\left(\rho_{c, e}+\rho_{c, h}\right)=1-S_{e}$ is a dimensionless metric of the voltage drop in an electron contact, and $S_{e}=$ $\rho_{c, h} /\left(\rho_{c, e}+\rho_{c, h}\right)=1-S_{h}$ is an dimensionless metric of the voltage drop in a hole contact. Considering both contacts in a device, we can rewrite Equation (8a) to define a full-device selectivity as:

$$
S_{t o t}=\frac{V_{o c}}{i V_{o c}}=\left(S_{h}^{l}+S_{e}^{r}-1\right)=\left(1-S_{e}^{l}-S_{h}^{r}\right) .
$$

Note that there is a conceptual difference between the preferred and the actual polarity of a contact and that they do not necessarily match: The preferred polarity depends on the contact alone and is defined by its selectivity, through Equation (8b). The actual polarity depends on the difference of selectivity between the two contacts, as expressed in Equations (8a-8c). In other words, the electron contact of a finished device is not defined by the absolute value of its selectivity but by a higher selectivity towards electrons than the other contact, as previously described by Roe et al. ${ }^{18}$ In a device with two contacts that are selective towards electrons, the contact with the lowest selectivity towards electrons will necessarily act as the hole contact, at the expense of $V_{o c}$. Such examples are presented in Section IV.

\section{F. Conductivity}

Unfortunately, Equations (6-8), which are valid at open circuit, cannot be extended to other operation points, and in particular to the maximum power point. Indeed, away from open circuit, the partial current densities $j_{e}$ and $j_{h}$ flowing through the partial specific contact resistances of a given contact no longer match, and the relationships between the resistances, the 
implied voltage, and the external voltage become more complex. However, the qualitative analysis remains true: a high sum of the electron and hole partial specific contact resistances, characteristic of a passivating contact, will lead to a high implied voltage at the maximum power point $\left(i V_{m p p}\right)$. Similarly, a high ratio of the electron and hole partial specific contact resistances, characteristic of a selective contact, will lead to full extraction of the cell's electrochemical potential at the maximum power point $\left(V_{m p p}=i V_{m p p}\right)$, although the linear relation from Equation (8b) is no longer valid.

An important difference from open circuit is resistive losses due to the flow of current through the cell. In the exemplary solar cell displayed in Figure 1, with a left hole contact and a right electron contact, this power loss amounts to:

$$
P_{\text {res,loss }}=\left(\rho_{c, h}^{l}+\rho_{c, e}^{r}\right) j_{m p p}^{2}
$$

with $j_{m p p}$ the total current density at the maximum power point. The higher the "majority-carrier" (carrier type intended to be collected) specific contact resistances, the lower $F F$ and the cell efficiency. This conductivity requirement means that, at each contact, the majority-carrier specific contact resistance must not only be smaller by orders of magnitude than the minoritycarrier specific contact resistance, but it also needs to be small in absolute value. In practice, assuming $j_{m p p} \approx j_{p h}$, $\left(\rho_{c, h}^{l}+\rho_{c, e}^{r}\right)<1 \mathrm{~W} \cdot \mathrm{cm}^{-2} /\left(10000 \times j_{p h}^{2}\right)$ ensures an absolute reduction in efficiency due to resistive losses of less than $0.1 \%$ (i.e. $P_{\text {res,loss }}<0.1 \mathrm{~mW} . \mathrm{cm}^{-2}$ ). For $j_{p h}=40 \mathrm{~mA} . \mathrm{cm}^{-2}$, as in excellent c-Si cells, this yields $\rho_{c, h}^{l}+\rho_{c, e}^{r}<62.5 \mathrm{~m} \Omega . \mathrm{cm}^{2}$; for $j_{p h}=20 \mathrm{~mA} \cdot \mathrm{cm}^{-2}$, as in c-Si-based tandems, the allowable sum quadruples to $250 \mathrm{~m} \Omega \cdot \mathrm{cm}^{2}$.

\section{G. Relationship between passivation, selectivity, and conductivity}

Importantly, for an individual contact, passivation $\left(\rho_{c, t o t}^{l}=\rho_{c, e}^{l}+\rho_{c, h}^{l} \gg 20 \Omega . \mathrm{cm}^{2}\right.$ and $\rho_{c, t o t}^{r}=\rho_{c, e}^{r}+\rho_{c, h}^{r} \gg 20 \Omega . \mathrm{cm}^{2}$ for c-Si cells with $\left.j_{p h} \approx 40 \mathrm{~mA} \cdot \mathrm{cm}^{-2}\right)$ and conductivity $\left(\rho_{c, h}^{l} \ll 1 \Omega . \mathrm{cm}^{2}\right.$ and $\rho_{c, e}^{r} \ll 1 \Omega$. $\mathrm{cm}^{2}$ for c-Si cells with $j_{p h} \approx$ $\left.40 \mathrm{~mA} \cdot \mathrm{cm}^{-2}\right)$ imply selectivity $\left(S_{e}^{l}=\rho_{c, h}^{l} /\left[\rho_{c, e}^{l}+\rho_{c, h}^{l}\right]=1-S_{h}^{l} \ll 1\right.$ and $S_{h}^{r}=\rho_{c, e}^{r} /\left[\rho_{c, e}^{r}+\rho_{c, h}^{r}\right]=1-S_{e}^{r} \ll 1$, leading to $S_{h}^{l} \approx 1$ and $S_{e}^{r} \approx 1$ ). However, at constant contact fraction, the reciprocal is not true. Numerous examples of selective yet poor full-area contacts exist. Even when combined with either passivation or conductivity, selectivity does not ensure high performance. For instance, for c-Si cells, aluminum back-surface field contacts have high selectivity and high conductivity but poor performance due to weak passivation. Assuming a saturation recombination current density of $550 \mathrm{fA} . \mathrm{cm}^{-2}-\mathrm{which}$ translates into an electron resistance of $\rho_{c, e}=16.2 \Omega \cdot \mathrm{cm}^{2}$ at open circuit—and a hole resistance of $\rho_{c, h}=5 \mathrm{~m} \Omega \cdot \mathrm{cm}^{2},{ }^{16}$ the ratio $V_{o c} / i V_{o c}>0.999$. The hole specific contact resistance is lower than the electron resistance by more than 3 orders of magnitude at open circuit, but both are low in absolute value. These cells thus tend to have relatively high $F F$ and $V_{o c}$ approaching $i V_{o c},{ }^{30}$ but $i V_{o c}$ itself is low $(<650 \mathrm{mV})$. 
Similarly, an amorphous silicon (a-Si:H) heterojunction hole contact to c-Si with thick intrinsic and doped a-Si:H layers is highly selective and passivating, enabling high $i V_{o c}$ and high $V_{o c}$, but its conductivity is poor, leading to low $F F$ and efficiency. Augusto et al. have demonstrated such a device having a saturation recombination current density of $0.1 \mathrm{fA} . \mathrm{cm}^{-2}, 31$ equivalent to a passivation metric of $\rho_{c, t o t}=1250 \Omega \mathrm{cm}^{2}$ at open circuit. Assuming an equal distribution of the resistances between both contacts (i.e. $\rho_{c, h}^{l}=\rho_{c, e}^{r}$ and $\rho_{c, e}^{l}=\rho_{c, h}^{r}$ )—a reasonable assumption, as shown by Lachenal et al. ${ }^{32}$-this corresponds to a summed majority-carrier contact resistance of $\rho_{c, h}^{l}+\rho_{c, e}^{r}=3.3 \Omega . \mathrm{cm}^{2}$. For this device, $V_{o c} / i V_{o c}>0.99$, yet the power losses due to series resistance in the contacts amounts to about $5 \%$ in absolute value.

This reasoning can also be reversed, and doing so reveals how partial specific contact resistances can be accessed from common device measurements. Consider a silicon heterojunction solar cell fabricated from a $150-\mu \mathrm{m}$-thick high-quality wafer with a bulk lifetime of $10 \mathrm{~ms}$. The maximum achievable $i V_{o c}$ of such a cell (i.e. with perfect passivation) is approximatively $750 \mathrm{mV}$. Due to imperfect passivation and selectivity, actual cells typically achieve $i V_{o c}=730 \mathrm{mV}$ and $V_{o c}=720 \mathrm{mV}$, and thus $V_{o c} / i V_{o c}=S_{t o t}=1-S_{e}^{l}-S_{h}^{r}=0.986$. Also, per Equation (6), this $i V_{o c}$ corresponds to a full-device passivation metric of $\rho_{c, t o t}=31.1 \Omega \cdot \mathrm{cm}^{2}$. Assuming again that the resistances distribute equally between the two contacts, $\rho_{c, h}^{l}+\rho_{c, e}^{l}=\rho_{c, h}^{r}+$ $\rho_{c, e}^{r}=2 \times \rho_{c, t o t}=62.2 \Omega \cdot \mathrm{cm}^{2}$ and $S_{h}^{l}=S_{e}^{r}=1-\left(\left(1-S_{t o t}\right) / 2\right)=0.993$. Thus, we can calculate the majority- and minority-carrier specific contact resistances at open circuit: $\rho_{c, h}^{l}=\rho_{c, e}^{r}=\left(1-S_{h}^{l}\right) \times\left(\rho_{c, h}^{l}+\rho_{c, e}^{l}\right)=\left(1-S_{e}^{r}\right) \times\left(\rho_{c, h}^{r}+\right.$ $\left.\rho_{c, e}^{r}\right)=0.44 \Omega \cdot \mathrm{cm}^{2}$ and $\rho_{c, e}^{l}=\rho_{c, h}^{r}=61.8 \Omega \cdot \mathrm{cm}^{2}$, which translate into a saturation recombination current density of $6.4 \mathrm{fA} . \mathrm{cm}^{-2}$. These results closely match the typical values reported in the literature ${ }^{15,33}$ and predict a cell with an efficiency above $20 \%$.

Therefore, at constant contact fraction, selectivity is necessary but not sufficient to ensure high cell efficiency. Thus, although selectivity is an intuitive concept to understand the inner working of contacts, it is not a meaningful metric for most cases. On the other hand, combined passivation and conductivity are necessary and sufficient for optimal contact performance. However, as demonstrated by Brendel et al., ${ }^{16,26}$ if both majority-carrier and minority-carrier specific contact resistances can be scaled arbitrarily by adjusting the contact fraction $f_{c}$-i.e. the partial contact resistances of the full device, in $\Omega$, are linear functions of $f_{c}$ (spreading resistance is neglected) — then a high enough ratio of the specific contact resistances (in $\Omega . \mathrm{cm}^{2}$ ) coupled with an optimal $f_{c}$ can simultaneously provide passivation and conductivity, leading to high efficiency. That is, provided optimal contact fraction, selectivity defines the limiting efficiency of a cell imposed by a contact (this optimal contact fraction, in turn, can be obtained from $j_{0, c}-\rho_{c}$ contour plots). ${ }^{15,26}$ Only under this assumption of an arbitrary contact fraction is 
a "carrier-selective" contact what is truly desired. However, scaling the partial specific contact resistances by adjusting the contact fraction has physical limits: for highly resistive absorbers or small contact fractions, spreading resistance cannot be neglected; for contact structures with high majority-carriers resistance, the contact fraction cannot exceed the cell area (modulo a surface geometry factor that is itself limited).

\section{PC1D SIMULATION DETAILS}

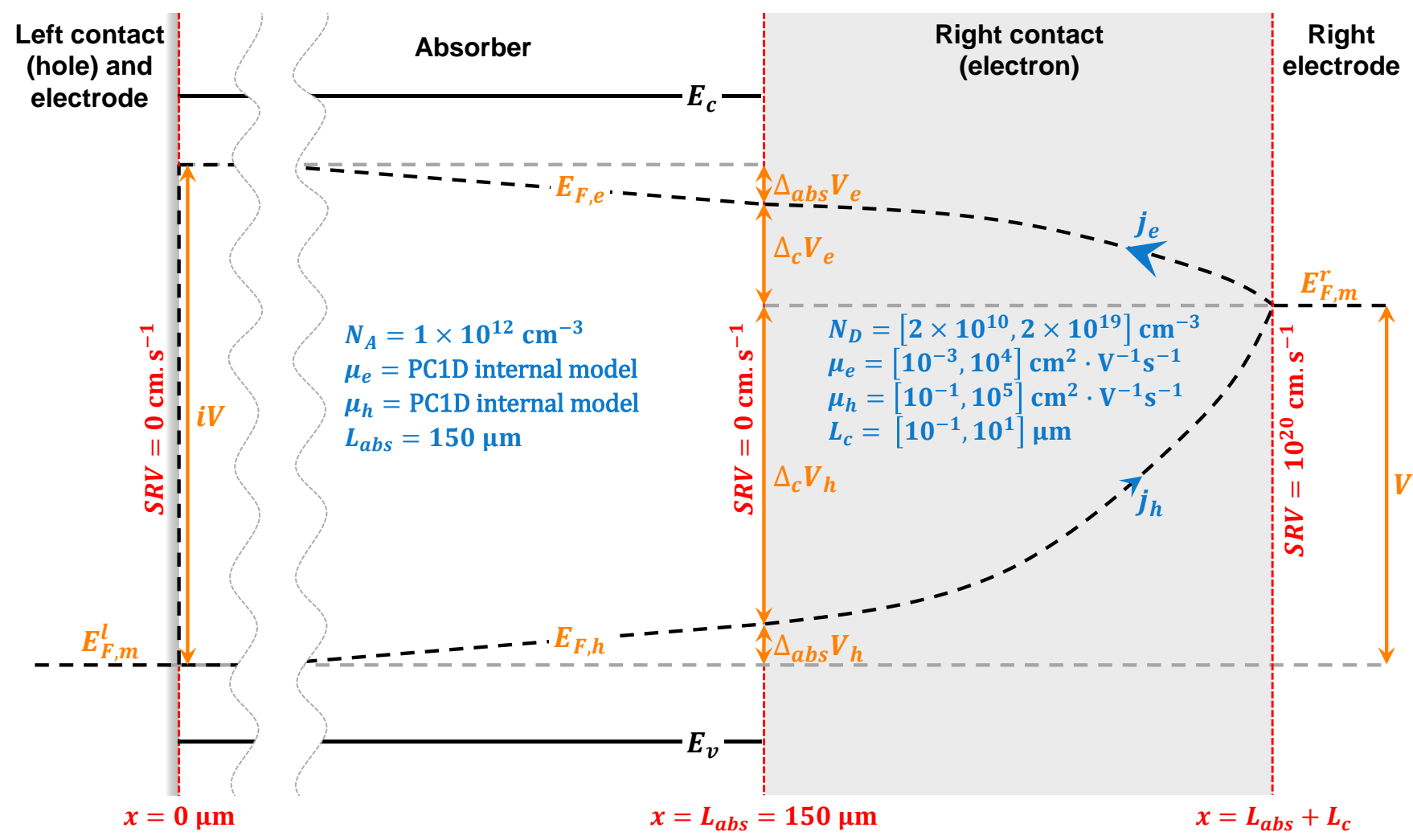

FIG. 2. Basic structure of the simulated device, with key parameters shown. The left (hole) contact is assumed to be infinitesimally thin and perfect, with no voltage drop. The SRV at this interface, as well as at the right absorber/contact interface, is set to $0 \mathrm{~cm} . \mathrm{s}^{-1}$. The SRV at the right (electron) contact/electrode interface, however, is set to $10^{20} \mathrm{~cm} \cdot \mathrm{s}^{-1}$, equivalent to an infinite SRV, as the metallic electrode cannot sustain separate qFLs. The doping density $N_{D}$, carrier mobilites $\mu_{e / h}$, and contact thickness $L_{c}$ are varied logarithmically in the right (electron) contact. Brackets specify the bounds of the variation explored for each parameter. From the amplitude of the qFL variations in the contact and the absorber, we obtain voltage drops $\Delta_{c} V_{e / h}$ and $\Delta_{a b s} V_{e / h}$, respectively. The current densities $j_{e / h}$ are partial current densities in the electron contact and are constant throughout its thickness, as generation and recombination are assumed to be null within the contact.

In order to validate our analytical model, we utilize PC1D to simulate a c-Si homojunction solar cell. While the model is not restricted to a particular material, c-Si is convenient for ease of simulation and comparison to known experimental trends.

As shown in Figure 2, the absorber of the test cell is a p-type, 150- $\mu \mathrm{m}$-thick wafer, with a doping density of $10^{12} \mathrm{~cm}^{-3}$ and a bulk lifetime of $10 \mathrm{~ms}$ so that recombination is dominated by Auger and radiative processes. The cell is illuminated from the left by monochromatic light with a wavelength of $1000 \mathrm{~nm}$; along with internal reflections at the front and back surfaces, this ensures a constant generation rate throughout the absorber with a total photocurrent density of $39.25 \mathrm{~mA} . \mathrm{cm}^{-2}$. Auger and radiative recombination are simulated in the bulk of the absorber through PC1D internal models, while recombination at the 
left and right absorber/contact interfaces is turned off by specifying a surface recombination velocity (SRV) of $0 \mathrm{~cm} . \mathrm{s}^{-1}$. This is equivalent to perfect surface passivation of the c-Si absorber. The left contact is modelled as an infinitesimally thin perfect hole contact, located at $x=0$, with no recombination or resistive losses. Thus, the $\mathrm{qFL}$ collapse in the left contact occurs abruptly at the contact/electrode interface and entirely within the electron qFL; the qFL of holes is constant across the interface. The right electron contact has a thickness ranging from 0.1 to $10 \mu \mathrm{m}$ and a donor doping density of $2 \times 10^{10}$ to $2 \times 10^{19} \mathrm{~cm}^{-3}$. The electron mobility ranges from $10^{-3}$ to $10^{4} \mathrm{~cm}^{2} \cdot \mathrm{V}^{-1} \cdot \mathrm{s}^{-1}$ and the hole mobility from $10^{-1}$ to $10^{5} \mathrm{~cm}^{2} \cdot \mathrm{V}^{-1} \cdot \mathrm{s}^{-1}$. These mobility combinations are not representative of silicon or any other known materials; the goal is to allow for the widest possible range of electron and hole conductivities without the constraints of realistic materials. The four right-contact parameters are varied logarithmically —in 7 steps for the electron mobility, 6 steps for the hole mobility, 5 steps for the thickness, and 50 steps for the doping density-leading to 10,500 different cases. Absorption is disabled within the right contact, and recombination is limited to the contact/electrode interface where the (actual, not effective) SRV is set to $10^{20} \mathrm{~cm} \cdot \mathrm{s}^{-1}$, rendering a close-toinfinitely-fast surface recombination process. Thus, electron-holes pairs can only be generated within the absorber and can then either be extracted, recombine within the bulk of the absorber, or recombine at the right contact/electrode interface.

For each of the 10,500 simulated cases, we extract band diagrams and partial current densities at the maximum power point and open circuit - in particular the partial current densities at the interface between the absorber and the electron contact $\left(x=L_{a b s}\right)$. The absence of generation and recombination leads to constant partial current densities within the contact, which are equal to these partial current densities at the absorber/contact interface. The current density flowing out of the cell is the difference between these partial electron and hole current densities, and is equal to zero at open circuit (i.e. $\left.j_{e}=j_{h}\right)$. The implied voltage $(i V)$ of the cell is calculated from the qFL separation at the left absorber/contact interface $(x=0)$, the external voltage $(V)$ is determined from the difference between the unique Fermi level at the right contact/electrode interface $(x=$ $\left.L_{a b s}+L_{c}\right)$ and the unique Fermi level at the left contact/electrode interface $(x=0)$. We calculate partial voltage drops across the electron contact $\left(\Delta_{c} V_{e / h}\right)$ from the difference between the qFLs at the right absorber/electron interface $\left(x=L_{a b s}\right)$ and the Fermi level at the right contact/electrode interface $\left(x=L_{a b s}+L_{c}\right)$. While the qFL collapse is ideally limited to within the contact, this is not generally the case in actual devices and in our PC1D simulations. We account for any reduction in qFL separation within the absorber by similarly extracting partial voltage drops $\Delta_{a b s} V_{e / h}$ across the absorber, measured from the left contact/absorber interface ( $x=0$, at which $i V$ is measured) to the right absorber/contact interface $\left(x=L_{a b s}\right)$.

The partial specific contact resistances $\rho_{c, e / h}$ are obtained by dividing the partial voltage drops $\Delta_{c} V_{e / h}$ across the contact by the partial current densities $j_{e / h}$ flowing through it, per Equation (3). This calculation works because, as mentioned above, the current density is constant in the contact due to the absence of generation and recombination. However, as part of the qFL 
collapse can occur in the absorber, we must also account for its resistance contribution, which we do by introducing partial specific absorber resistance terms $\rho_{a b s, e / h}$. These terms are calculated by dividing $\Delta_{a b s} V_{e / h}$ by the partial current densities $j_{e / h}$ flowing out of the absorber at $x=L_{a b s}$. The partial specific total resistances $\rho_{\text {tot }, e / h}$ are then the sum of the partial specific contact and absorber resistances: $\rho_{t o t, e / h}=\rho_{c, e / h}+\rho_{a b s, e / h}$. It should be noted that the partial specific absorber resistance terms calculated here are not true absorber resistance terms, as the partial current densities $j_{e / h}$ are not representative of the current densities throughout the absorber. Rather, $\rho_{a b s, e / h}$ are equivalent partial specific resistances, representing the contribution of the absorber to the partial specific resistances of the device. More specifically, they are the average partial resistances of the absorber weighted by the amount of current going through it at each position, as detailed in Appendix A.

Not all of the 10,500 simulated cases input into PC1D converge to a solution. As a result, the data must be filtered to remove invalid solutions. First, the partial current densities at the maximum power point at the right absorber/contact interface are summed and compared to the $j_{m p p}$ determined by PC1D. Simulations in which the two values are not close or exact matches $( \pm 0.1 \%)$ are discarded. Similarly, the partial current densities at open circuit at the right absorber/contact interface are summed, and values which are not close to or exactly zero are discarded. This reduces the final number of data points: we report 6,935 cases at open circuit, at which there are many non-converging solutions, and 9,402 cases at the maximum power point.

\section{RESULTS AND DISCUSSION}

We simulated the current density-voltage characteristics of 10,500 silicon solar cells that were identical save for their fullarea rear electron contacts, which had varying donor doping densities, electron and hole mobilities, and thicknesses, and thus varying partial specific contact resistances, $\rho_{c, e / h}$. For each cell, we recorded its $i V, V$, and $\rho_{c, e / h}$ and $\rho_{a b s, e / h}$ at open circuit and at the maximum power point, as well as its efficiency. In addition to the results and figures presented in this section, band diagrams of cells displaying characteristic behaviors in terms of passivation, selectivity, and conductivity are shown in the Supplementary Material (Figure S2), along with the positions of these characteristic cells in reproductions of Figures 3, 4, and 5 (Figure S3).

\section{A. Open circuit: implied voltage and passivation}


a) $\begin{array}{lllll} & \mathrm{iV}_{\mathrm{oc}}(\mathrm{mV}) \\ 450 & 500 & 550 & 600 \quad 650 \quad 700 \quad 750\end{array}$
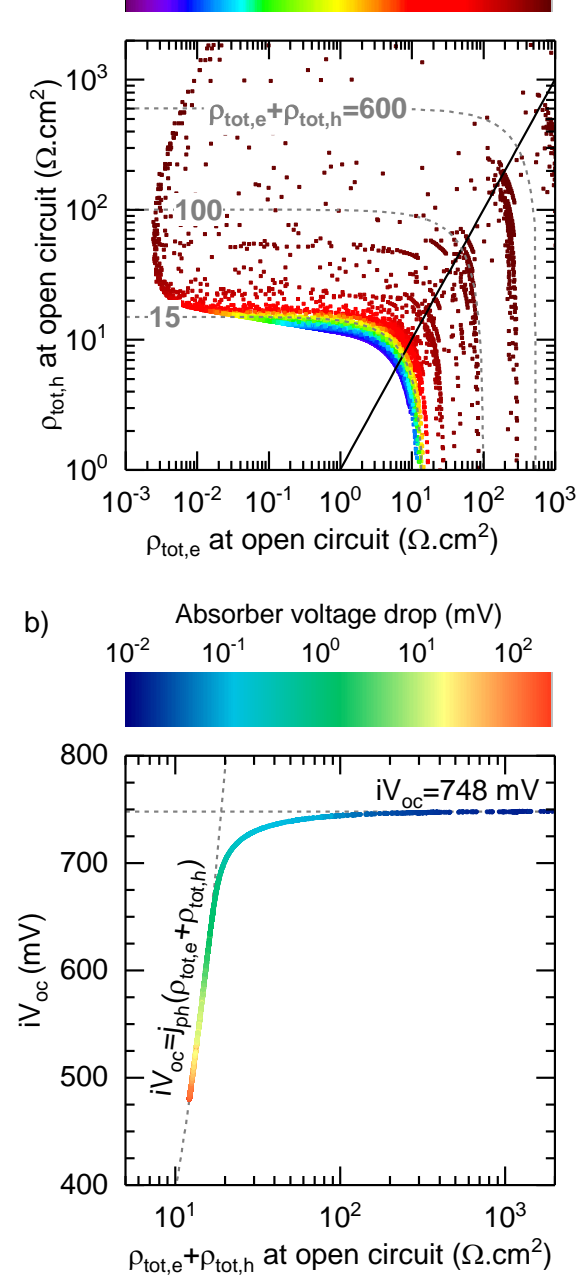

FIG. 3. Implied open-circuit voltages from PC1D simulations of 10,500 silicon solar cells; each point corresponds to one simulated cell. (a) $i V_{o c}$ as a function of the hole and electron specific total resistances at open circuit. $\rho_{t o t, e}=\rho_{t o t, h}$ is displayed as a continuous black line, and constant sums of the partial resistances $\rho_{t o t, e}+\rho_{t o t, h}$ are shown as dashed gray lines. (b) The same $i V_{o c}$ data displayed as a function of the sum of the electron and holes specific total resistances. Limiting behaviors, at low and high $\rho_{t o t, e}+\rho_{t o t, h}$, are displayed with dashed gray lines. The logarithmic color scale indicates the voltage drop occurring within the absorber.

Figure 3a shows the dependence of $i V_{o c}$ on the electron and hole specific total resistances, $\rho_{t o t, e / h}=\rho_{c, e / h}+\rho_{a b s, e / h}$. Results when omitting the contribution of the absorber to the partial specific resistances can be found in the Supplementary Material (Figure S4). As expected, $i V_{o c}$ values are symmetric with respect to the $\rho_{t o t, e}=\rho_{t o t, h}$ line: the qFL separation is independent of the preferred or actual polarity of the contact (discussed in Section II-E), as only its passivating capability counts. Interestingly, the minimum $i V_{o c}$ value achievable is approximately $480 \mathrm{mV}$ and the sum of the resistances is always higher than $10 \Omega . \mathrm{cm}^{2}$. This is due to the inherent absorber resistance, which, in the case of a contact that is very conductive to both electrons and holes, causes a non-negligible fraction of the qFL collapse to happen in the region of the absorber adjacent to the contact. In this region, the densities of electron and holes will be reduced, leading to an increase in resistivity. As a result, 
the partial specific resistances of the absorber dominate over the resistances of the contact, and the sum of the partial specific total resistances has a lower bound of $12.2 \Omega . \mathrm{cm}^{2}$ at open circuit for the nearly intrinsic wafer considered here.

In Figure $3 b$, the same $i V_{o c}$ data are displayed as a function of the sum of the partial specific total resistances- the passivation metric identified in Equation (7b), but inclusive of the absorber resistance contributions. As expected from the discussion in Section II, $i V_{o c}$ is a function of this sum according to Equation (6). That is, Equation (6)—again with the contact resistances replaced by total resistances to account for the absorber - provides an exact fit to the data generated by PC1D. Two particular regimes can be identified. When $\rho_{t o t, e}+\rho_{t o t, h}<20 \Omega . \mathrm{cm}^{2}$, recombination at the contact/electrode interface dominates and $i V_{o c}$ depends linearly on the sum of the resistances, with a proportionality factor equal to $j_{p h}$. Conversely, when $\rho_{\text {tot }, e}+\rho_{\text {tot }, h}>100 \Omega \mathrm{cm}^{2}$, recombination at the contact/electrode interface is effectively curtailed and radiative and Auger recombination in the absorber dominate, causing $i V_{o c}$ to approach a plateau of $748 \mathrm{mV}$. When $\rho_{\text {tot,e }}+\rho_{\text {tot,h }}>$ $1000 \times\left((2 / 3) k_{B} T\right) /\left(q j_{p h}\right)=440 \Omega . \mathrm{cm}^{2}$, the maximal $i V_{o c}$ is effectively reached. The role of the absorber resistance in maintaining the qFL separation is apparent on the color scale, especially when the contact passivation is insufficient: the qFL collapse, or voltage drop, happens increasingly within the absorber and can reach over $460 \mathrm{mV}$.

\section{B. Open circuit: external voltage and selectivity}



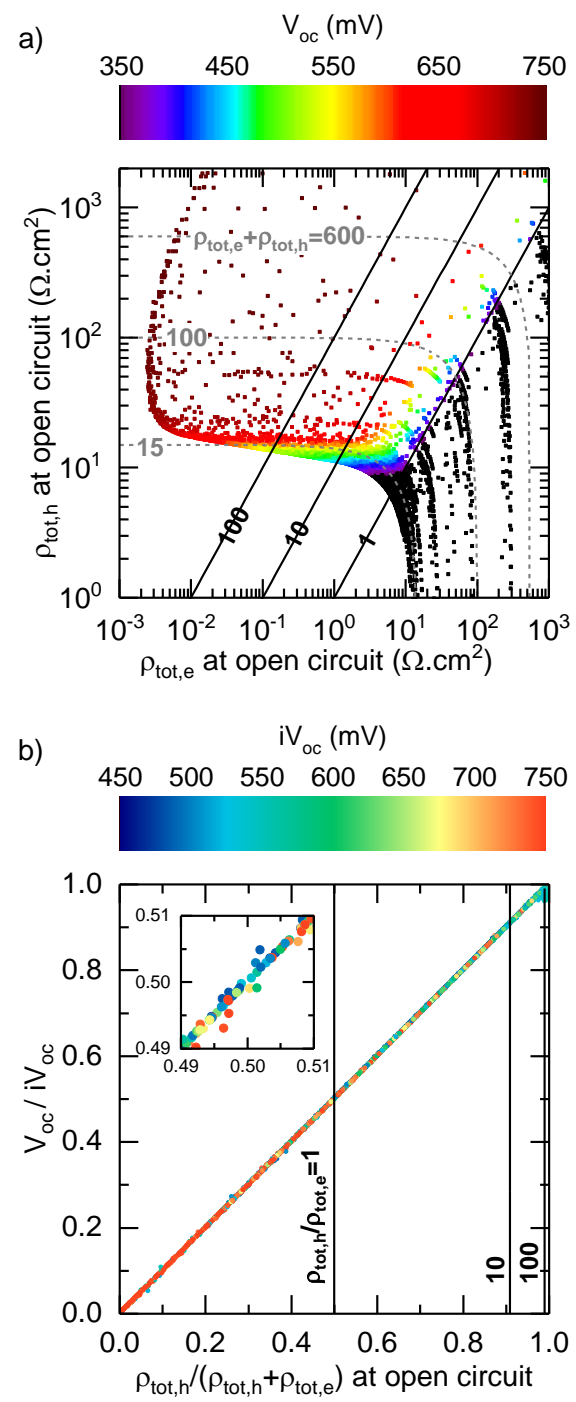

FIG. 4. Open-circuit voltages from PC1D simulations of 10,500 silicon solar cells; each point corresponds to one simulated cell. (a) $V_{o c}$ as a function of the hole and electron specific total resistances at open circuit. The color scale is limited to $350 \mathrm{mV}$ for the sake of clarity and black points indicate lower $V_{o c}$ values. Constant ratios of partial resistance $\rho_{t o t, h} / \rho_{t o t, e}$ (equal to 1,10, and 100) are displayed as continuous black lines, and constant sums of the partial resistances $\rho_{t o t, e}+\rho_{t o t, h}$ are shown as dashed gray lines. (b) The $V_{o c} / i V_{o c}$ ratio for these same data points displayed as a function of the selectivity metric $\rho_{t o t, h} /\left(\rho_{t o t, e}+\rho_{t o t, h}\right)$. Constant ratios of the partial resistances $\rho_{\text {tot }, h} / \rho_{\text {tot }, e}$ are displayed as vertical continuous black lines. The color scale indicates $i V_{o c}$ for each data point. The inset shows a magnified region of the graph and uses the same $i V_{o c}$ color scale.

Figure 4a is similar to Figure 3a but displays $V_{o c}$ instead of $i V_{o c}$. A similar Figure, displaying the $V_{o c} / i V_{o c}$ ratio is presented in the Supplementary Material (Figure S5). A strong asymmetry is now evident across the $\rho_{\text {tot }, e}=\rho_{\text {tot }, h}$ line: In order to obtain $V_{o c}>374 \mathrm{mV}$, the hole resistance must be higher than the electron resistance. The position of a contact relative to the $\rho_{\text {tot }, e}=$ $\rho_{\text {tot }, h}$ line defines its preferred polarity: contacts left of the line are more selective towards electrons, whereas contacts right of the line are more selective towards holes. These cases are remarkable examples of how doping alone does not define the preferred polarity of a contact, as, in this study, the contact is consistently n-type. Even with non-negligible n-type doping- 
as high as $1.2 \times 10^{15} \mathrm{~cm}^{-3}$-a strong selectivity towards holes $\left(S_{h}=1-S_{e}=\rho_{\text {tot }, e} /\left(\rho_{\text {tot }, e}+\rho_{\text {tot }, h}\right)>0.8\right)$ is achievable if the ratio of mobilities is high enough. These results further illustrate the concept of a "mobility-junction cell" described by Würfel et al. ${ }^{6}$ and demonstrate that such a cell can be successfully implemented in a simple software program such as PC1D. However, as previously discussed in Section II-E, the actual and preferred polarities do not necessarily match. In this example, contacts right of the $\rho_{t o t, e}=\rho_{t o t, h}$ line are still the electron contact of the device although they are more selective towards holes, as the left hole contact is assumed perfect and thus has a higher selectivity towards holes in any case.

In Figure $4 \mathrm{~b}$, the ratio $V_{o c} / i V_{o c}$ is shown as a function of the selectivity metric $S_{e}=\rho_{t o t, h} /\left(\rho_{t o t, e}+\rho_{t o t, h}\right)$, which appeared in Equation (8b), but this time with the absorber resistance contributions. The two ratios are identical (unity slope in Figure 4b), thus demonstrating the voltage-divider behavior described in Section II and validating the selectivity metric we propose. And, as evident from the data's color — which displays the corresponding $i V_{o c}$ for each point —and particularly the magnified inset, a high selectivity does not necessarily lead to excellent passivation, and vice-versa. Selectivity and passivation are independent contact properties, although the lack of one or the other leads to a poor $V_{o c}$.

\section{Maximum power point: efficiency and conductivity}




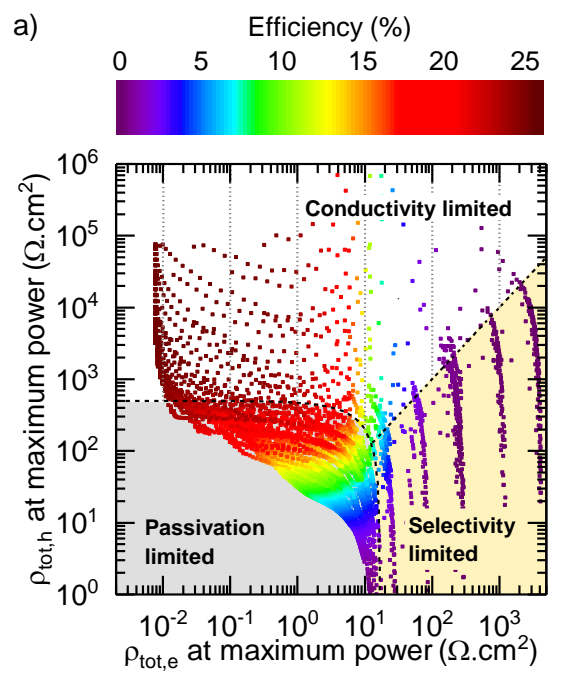

b) $\quad \rho_{\text {tot, } \mathrm{h}}$ at maximum power $\left(\Omega . \mathrm{cm}^{2}\right)$

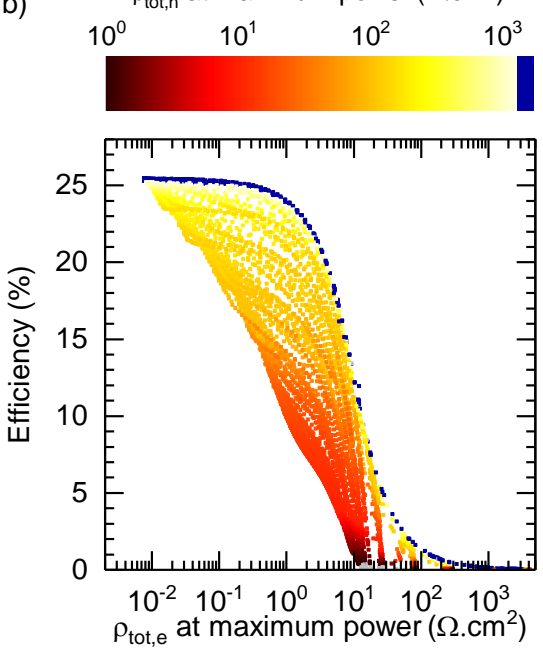

c) $\rho_{\text {tot, },} /\left(\rho_{\text {tot, }}+\rho_{\text {tot, }}\right)$ at maximum power $\left(\Omega . \mathrm{cm}^{2}\right)$
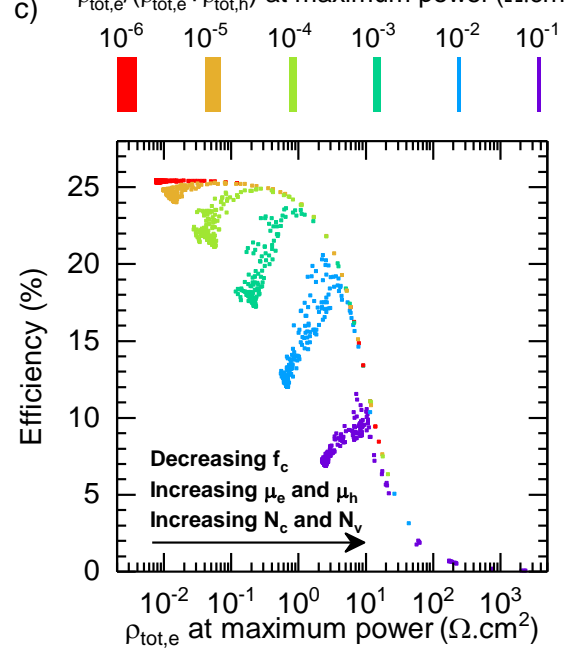

FIG. 5. Efficiencies from PC1D simulations of 10,500 silicon solar cells; each point corresponds to one simulated cell. (a) Efficiency as a function of the hole and electron specific total resistances at the maximum power point. Three regions are delineated where the efficiency is limited by passivation, selectivity, and conductivity. Constant electron resistances $\rho_{\text {tot,e }}$ are shown in dotted gray lines. (b) Efficiency as a 
function of the specific electron resistance $\rho_{\text {tot }, e}$, with a color scale that indicates the specific hole resistance $\rho_{\text {tot }, h}$. Contacts with $\rho_{\text {tot }, h}>$ $2000 \Omega \cdot \mathrm{cm}^{-2}$ are marked in dark blue. (c) Subset of Figure $5 \mathrm{~b}$ highlighting, with the color scale, groups of iso-selectivity $1-S_{e}=S_{h}=$ $\rho_{\text {tot }, e} /\left(\rho_{\text {tot }, e}+\rho_{\text {tot }, h}\right)$ contacts. These are projections of oblique slices from Figure $5 \mathrm{a}$.

Figure 5a displays the efficiency of the same simulated solar cells that appear in Figures 3 and 4 . Additional metrics $\left(i V_{m p p}\right.$, $V_{m p p}, V_{m p p} / i V_{m p p}$, and $j_{m p p}$ ) are similarly displayed in the Supplementary material (Figure S6). Note that, as the efficiency is calculated at the cells' maximum power point, the partial specific total resistances on the $\mathrm{x}$ - and $\mathrm{y}$-axes are those at the maximum power point instead of at open circuit. These resistances are thus different from those in Figures $3 \mathrm{a}$ and $4 \mathrm{a}$ and the axes are scaled accordingly. Note, however, that the electron specific total resistance tends to be similar at the maximum power point and open circuit; the electron density in the contact is usually dominated by intentional extrinsic doping rather than by the flow of photo-generated excess carriers from the absorber, and thus the associated resistance changes little with $i V$. On the other hand, the hole resistance increases by up to three orders of magnitude in transitioning from open circuit to the maximum power point, as the hole density is in most cases dictated by the flow of excess carriers from the absorber, which itself depends on the point of operation of the cell. Contrary to $i V_{o c}$ and $V_{o c}$ explored above, the efficiency is not uniquely defined by the partial specific total resistances $\left(\rho_{\text {tot }, e}, \rho_{\text {tot }, h}\right)$. This is visible in Figure 5a as colors bleeding into one another: data points with similar $\rho_{t o t, e}$ and $\rho_{t o t, h}$ can have different efficiencies, especially for $10^{-2} \Omega . \mathrm{cm}^{2}<\rho_{\text {tot }, e}<10^{1} \Omega . \mathrm{cm}^{2}$ and $10^{1} \Omega . \mathrm{cm}^{2}<\rho_{t o t, h}<$ $10^{3} \Omega . \mathrm{cm}^{2}$. This result is not surprising in light of the voltage divider model presented in Figure 1: Unlike at open circuit, current flows out of the voltage divider at the maximum power point, and thus the contributions to the voltage drop from the resistors are no longer equally proportional to their respective resistances values. Instead, their contributions are proportional to their partial current density-resistance products. These partial current densities, in their turn, depend on the injection level at maximum power point, which can vary from cell to cell as shown by Descoeudres et al.. ${ }^{34}$ In point of fact, in a threedimensional rendering of Figure 5a that includes the cell current density $\left(\rho_{t o t, e}, \rho_{t o t, h}, j_{m p p}\right.$ axes), the functional unicity of the efficiency is recovered, as demonstrated in the Supplementary Material (Figures S7-S8).

Nevertheless, three regions can be identified in Figure 5a. In shaded gray, in the bottom left corner, the efficiency is limited by poor passivation, as the partial-current-density-weighted sum of the resistances is too low. In the bottom right corner, the efficiency is limited by poor (electron) selectivity: the ratio between the electron and hole resistances is low, leading to a low $V_{m p p} / i V_{m p p}$ ratio. Finally, in the upper part of the graph (non-shaded region), the efficiency is limited by the conductivity of majority carriers. In this area, the efficiency is solely dictated by the value of the electron (i.e. majority carrier) resistance, and the iso-electron-resistance lines, shown in dotted gray, are also iso-efficiency lines. This behavior is highlighted in Figure 5b, which shows the cell efficiency as a function of the electron specific total resistance. For most cells with low hole specific total 
resistance $\left(\rho_{t o t, h}<2000 \Omega . \mathrm{cm}^{2}\right)$, displayed with maroon to yellow dots, no functional relationship can be established between the electron specific total resistance and the efficiency. However, for cells with high hole specific total resistance $\left(\rho_{\text {tot }, h}>\right.$ $2000 \Omega . \mathrm{cm}^{2}$ ), shown in dark blue, the efficiency is a unique, monotonic function of the electron specific total resistance.

Strikingly, for the constant-contact-fraction case considered thus far, selectivity dictates efficiency only in a restricted portion of the defined partial-specific-total-resistance parameter space (bottom-right in Figure 5a), and the cell efficiency is low in this region. Conversely, high efficiencies can be achieved only with a high hole specific total resistance and a low electron specific total resistance, and their exact ratio is unimportant, consistent with the finding from Section II that selectivity is not a relevant metric. However, if we lift the constraint of unity contact fraction and neglect spreading resistance, the electron and hole specific contact resistances can be varied together by changing the contact fraction, while their ratio remains constant. That is, varying the contact fraction corresponds to navigating along iso-selectivity lines, $\rho_{t o t, h}=\left(S_{e} /\left(1-S_{e}\right)\right) \rho_{t o t, e}$ with $S_{e}$ constant, which extend southwest to northeast in Figures 3a, 4a, and 5a. The black lines in Figure 4a are such iso-selectivity lines. Likewise, varying the electron and hole mobilities in concert, or their effective densities of states in concert, while keeping all other parameters unchanged, also corresponds to moving along these lines.

Figure $5 \mathrm{c}$ shows a subset of the data in Figure 5b, divided into separate groups of comparable selectivity. Figure 5c is, thus, a projection of oblique slices of Figure 5a, as shown in Figure S10. Moving along the x-axis within each group of constant selectivity points in Figure 5c is equivalent to changing the contact fraction, as adjusting the contact fraction scales both partial specific resistances $\rho_{\text {tot }, e}$ and $\rho_{\text {tot }, h}$ proportionally without altering the selectivity defined by the ratio $\rho_{\text {tot }, e} /\left(\rho_{\text {tot }, e}+\rho_{\text {tot }, h}\right)$. As demonstrated by Brendel et al., ${ }^{16}$ for each selectivity there is a unique value of $\rho_{t o t, e}$-corresponding, for a given contact, to a unique contact fraction - that yields maximum cell efficiency. The difference is that our generalized formalism does not lead to a unique functional relationship but to a family of solutions that converges to a unique function with increasing electron specific total resistance $\rho_{\text {tot,e }}$. Alternatively, if movement along the iso-selectivity lines is accomplished by varying the mobilities in concert instead of the contact fraction, the same results arises: there is an optimal (and finite) pairing of mobilities to achieve maximal efficiency, in agreement with several studies from the organic photovoltaics community. ${ }^{19,20,35,36}$ The same conclusion can also be drawn for varying effective densities of states. The latter insights are, however, of lesser practical value than that associated with contact fraction because mobilites and effective densities of states cannot independently be modified, as they are both strongly correlated to the charge carriers' effective masses. ${ }^{37}$

\section{CONCLUSION}

We presented a generalized analytical model of contacts to solar cells based on partial specific resistances. Using these electron and hole specific resistances, we proposed a set of metrics for passivation, conductivity, and selectivity that are 
consistent with 10,500 PC1D simulations. Although we used a silicon solar cell with a silicon homojunction contact for these simulations, the model can be applied to a wide range of absorber materials and contact structures, provided that the following assumptions are true:

- The device is in steady-state (no transient currents),

- Two separate quasi-Fermi levels, corresponding to positive and negative charge carriers, are allowed in the absorber and contacts,

- Only one Fermi level is allowed in the metallic electrodes,

- The partial electron and hole current densities follow the conductivity equations pointed out by Würfel et al. ${ }^{1,6}$ and are equal, at each point in space, to the product of their respective quasi-Fermi level gradients and their respective conductivities (the case of tunneling contacts can be handled by considering similar conductivity equations over the full thickness of the tunneling region).

This framework revealed that, at fixed contact fraction — as is the case with full-area contacts — combined passivation and conductivity are necessary and sufficient for optimal contact performance and imply selectivity. Selectivity is thus necessary, but not sufficient, for high cell efficiency, even when combined with either passivation or conductivity. However, when the contact fraction can be arbitrarily adjusted, selectivity drives the maximal efficiency of the device, as described by Brendel $e t$ $a l .{ }^{16}$ Nevertheless, in many cases, the assumption that the contact fraction can be scaled arbitrarily to achieve maximum efficiency is not realistic, as real contacts have a relatively narrow range of practical areal fraction: at the low end, contact fractions below $1 \%$ will lead to non-negligible series resistances; at the high end, exceeding $170 \%$ contact fraction, even when taking into account extra surface area from texturing, is impractical. Our study shows that some contact structures that would have a high selectivity, leading to $V_{o c}=i V_{o c}$, are not actually desirable.

For a particular contact structure, experimental measurement of the electron and hole specific contact resistances or specific total resistances is, however, not straightforward. Accessing the minority-carrier specific resistance, in particular, can be complex. As we have shown, in the case of an ideal absorber with perfect surface passivation, $i V_{o c}$ is a function of the sum of the specific total resistances, which act as a shunt resistance of the absorber. However, in practical cases (non-perfect surface passivation at the absorber/contact interface or non-negligible bulk defects within the absorber or the contact), parallel recombination processes can also be modelled as implied-voltage-dependent shunt resistances, making difficult the deconvolution between recombination at the contact/electrode interface and other sources of surface or bulk recombination. Comparing injection-dependent lifetimes (or implied- $J-V$ curves) before and after deposition of the electrode may offer a pathway, although deposition of the electrode must not degrade the passivation of the absorber for this to work; such 
degradation has been observed in c-Si heterojunction devices. ${ }^{38}$ Comparing $V_{o c}$ and $i V_{o c}$ is another approach, although the ratio $V_{o c} / i V_{o c}$ of a finished device is a function of both contacts' selectivities. Consequently, this method will work only when the selectivity of one contact is near perfect while the selectivity of the other contact is limited and, hence, fully responsible for the difference between $V_{o c}$ and $i V_{o c}$. Further work is thus required to establish robust techniques to measure minority-carrier specific resistance. Fortunately, $j_{0, c}$ extracted from lifetime measurements ${ }^{12}$ and $\rho_{c}$ extracted from transfer length measurements ${ }^{13}$ are useful proxies, though it is important to note that they are not usually measured at the MPP of the solar cell and that $j_{0, c}$ cannot be determined at all for many cases, including a wide range of heterojunction contacts. Hence, for now and until techniques are available to measure minority-carrier specific resistances, we recommend the use of $j_{0, c}-\rho_{c}$ contour plots, as popularized by Bullock and Cuevas, ${ }^{15,39}$ to visualize and compare the performance of different types of contacts, especially when the contact fractions is fixed.

\section{SUPPLEMENTARY MATERIAL}

The Supplementary Material includes an extension of the model to cells with non-negligible recombination at the absorber/contact interface. In addition, band diagrams of cells presenting characteristic behaviors are displayed, along with reproductions of Figure 3, 4, and 5 with the positions of the cells in question. Furthermore, we present results at open circuit when omitting the contribution of the absorber to the overall partial specific resistances. The ratio $V_{o c} / i V_{o c}$ as a function of the partial specific total resistances at open circuit is also displayed. Similarly, additional metrics at maximum power point $\left(i V_{m p p}\right.$,

$V_{m p p}, V_{m p p} / i V_{m p p}$, and $\left.j_{m p p}\right)$ are displayed as a function of the partial specific total resistances, also at maximum power point. 3D representations of the efficiency mapped as a function of both partial specific total resistances and the current density at maximum power point are shown, along with a demonstration of the functional unicity of the relationship between efficiency and $\left(\rho_{t o t, e}, \rho_{t o t, h}, j_{m p p}\right)$. Finally, we provide details on how Figure $5 \mathrm{c}$ is built from Figure 5a.

\section{ACKNOWLEDGEMENTS}

The information, data, or work presented herein was funded in part by the U.S. Department of Energy, Office of Energy Efficiency and Renewable Energy, under Award Number DE-EE0007552, Award Number DE-EE0008552, Award Number DE-EE00081567, and Award Number DE-EE00006335. Support was also provided by the Engineering Research Center Program of the National Science Foundation and the Office of Energy Efficiency and Renewable Energy of the Department of Energy under NSF Cooperative Agreement No. EEC-1041895, and by the National Science Foundation under award No. 1846685. The authors thank Michael Rienäcker, from ISFH, for restarting the conversation regarding this study, leading to the present publication. 


\section{APPENDIX A: FULL DERIVATION OF THE PARTIAL SPECIFIC CONTACT RESISTANCES}

To extend the general local relation from Würfel ${ }^{1}$ to a contact structure, we integrate the current-density equation over the thickness of the contact:

$$
\begin{aligned}
& \int_{L_{a b s}}^{L_{a b s}+L_{c}} j_{e}(x) d x=\frac{1}{q} \int_{L_{a b s}}^{L_{a b s}+L_{c}} \sigma_{e}(x) \frac{d E_{F, e}}{d x} d x, \\
& \int_{L_{a b s}}^{L_{a b s}+L_{c}} j_{h}(x) d x=\frac{1}{q} \int_{L_{a b s}}^{L_{a b s}+L_{c}} \sigma_{h}(x) \frac{d E_{F, h}}{d x} d x .
\end{aligned}
$$

Here, in agreement with Figure 2, $x=L_{a b s}$ corresponds to the inner edge of the contact, at the interface with the absorber, and $x=L_{a b s}+L_{c}$ corresponds to the outer edge of the contact, at the interface with the metallic electrode where only one $\mathrm{qFL}$ is allowed. The thickness of the contact is, thus, $L_{c}$.

As the qFLs as a function of position $E_{F, e / h}(x)$ are homeomorphisms - continually derivable bijections (otherwise the direction of the current within the contact could change) with continually derivable reciprocal functions across the width of the contact (otherwise infinite electric fields would be possible)—we can proceed with a change of variable in the integral: $d x=$ $\left(1 / \frac{d E}{d x}\right) d E$. Furthermore, as both generation and recombination are assumed to be null in the contact, the current density is constant through it $\left(j_{e / h}(x)=j_{e / h}\right.$ for $\left.L_{a b s}<x<L_{a b s}+L_{c}\right)$. As a result:

$$
\begin{aligned}
& j_{e} L_{c}=\frac{1}{q} \int_{\substack{E_{F, e}\left(x=L_{a b s}\right) \\
j_{F, h}\left(x=L_{a b s}+L_{c}\right)=E_{F, m}}}^{E_{F, e}\left(x=L_{a b s}+L_{c}\right)=E_{F, m}} \sigma_{e}(E) d E, \\
& j_{h} L_{c}=\frac{1}{q} \int_{E_{F, h}\left(x=L_{a b s}\right)} \sigma_{h}(E) d E .
\end{aligned}
$$

With this change of variable, the spatial dependence of the conductivities is replaced with a qFL dependence, and all of the spatial information is contained in the $L_{c}$ term. By introducing the average conductivities weighted by their qFL distributions, defined in Equations (4a-4b), we have:

$$
\begin{aligned}
& j_{e} L_{c}=\frac{\overline{\sigma_{e}}}{q}\left(E_{F, m}-E_{F, e}\left(x=L_{a b s}\right)\right)=\frac{\overline{\sigma_{e}}}{q} \Delta_{c} E_{F, e}=\overline{\sigma_{e}} \Delta_{c} V_{e}, \\
& j_{h} L_{c}=\frac{\overline{\sigma_{h}}}{q}\left(E_{F, m}-E_{F, h}\left(x=L_{a b s}\right)\right)=\frac{\overline{\sigma_{h}}}{q} \Delta_{c} E_{F, h}=\overline{\sigma_{h}} \Delta_{c} V_{h} .
\end{aligned}
$$

The partial specific contact resistances are then naturally defined as:

$$
\begin{gathered}
\rho_{c, e}=\frac{L_{c}}{\overline{\sigma_{e}}}, \\
\rho_{c, h}=\frac{L_{c}}{\overline{\sigma_{e}}} .
\end{gathered}
$$


The integration is still possible for the calculation of the partial specific contact resistances of the absorber, where the assumption of a constant current density is no longer valid. In the particular case where the difference between the generation rate $G(x)$ and the recombination rate $R(x)$ is constant in space, equal to $j_{p h} / L_{a b s}$ (with $L_{a b s}$ the width of the absorber), the left side of the equation becomes:

$$
\begin{aligned}
& \int_{0}^{L_{a b s}} j_{e}(x) d x=\int_{0}^{L_{a b s}} \int_{0}^{x}(G(y)-R(y)) d y d x=\int_{0}^{L_{a b s}} \int_{0}^{x} \frac{j_{p h}}{L_{a b s}} d y d x=\frac{j_{p h}}{2} L_{a b s}, \\
& \int_{0}^{L_{a b s}} j_{h}(x) d x=\int_{0}^{L_{a b s}} \int_{0}^{x}(G(y)-R(y)) d y d x=\int_{0}^{L_{a b s}} \int_{0}^{x} \frac{j_{p h}}{L_{a b s}} d y d x=\frac{j_{p h}}{2} L_{a b s} .
\end{aligned}
$$

Here, again in agreement with Figure 2, $x=0$ corresponds to the left absorber/contact interface and $x=L_{a b s}$ corresponds to right absorber/contact interface. As a result, the electron and hole partial specific resistance relations in Equations (A3a-A3b) have to include this $\frac{1}{2}$ term in the case of an absorber with spatially constant current generation:

$$
\begin{aligned}
& j_{p h} L_{a b s}=\frac{2 \overline{\sigma_{e}}}{q} \Delta_{a b s} E_{F, e}=2 \overline{\sigma_{e}} \Delta_{a b s} V_{e}, \\
& j_{p h} L_{a b s}=\frac{2 \overline{\sigma_{h}}}{q} \Delta_{a b s} E_{F, h}=2 \overline{\sigma_{h}} \Delta_{a b s} V_{h} .
\end{aligned}
$$

with the $\Delta_{a b s}$ operator indicating the qFL reduction or voltage drop across the absorber. Thus, the partial specific absorber resistances are:

$$
\begin{gathered}
\rho_{a b s, e}=\frac{L_{a b s}}{2 \overline{\sigma_{e}}}, \\
\rho_{a b s, h}=\frac{L_{a b s}}{2 \overline{\sigma_{h}}} .
\end{gathered}
$$

In these equations, the $\overline{\sigma_{e / h}}$ terms now refer to the (average) conductivities in the absorber.

It is to be noted that the specific absorber resistances calculated in Equations (A7a-A7b) are not the actual resistances to electrons and holes through the entirety of the absorber but are the equivalent resistances experienced on average by the charge carriers generated in the absorber. In more detail, they are the sums of the local resistances weighted by the amount of current going through them, as, in our example, the charge carriers generated at the front of the device must travel through the full absorber while the ones generated close to the back of the absorber only need to travel a short distance to reach the contact.

Regarding tunneling contacts, the analysis would appear to be inapplicable as Equation (1) predicts no current in (and, thus, through) the insulator. This implies that Equations (2-4) do not describe tunneling transport either. However, the tunneling barrier can be assigned equivalent specific contact resistances for electrons and holes, determined by the partial voltage drops and the partial current densities through it: $\rho_{t, e / h}=\Delta_{t} V_{e / h} / j_{e / h}$. Here the subscript " $t$ " indicates that the partial physical quantities (voltage drop and specific resistance) correspond to transport through the tunneling barrier. Breaking down the 
contributions from each layer of a given contact structure, considering band-edge transport when appropriate and tunneling when appropriate, and adding them as resistances in series, partial specific contact resistances can be calculated regardless of the presence of a tunneling layer. Hence, our framework can easily be generalized to tunneling contacts; Equation 5 in particular remains valid, even when tunneling is involved.

\section{APPENDIX B: APPROXIMATION OF THE MINIMAL SUM OF RESISTANCES TO ACHIEVE MAXIMAL iVoc}

Starting from Equation (6-7a) at open circuit, we have:

$$
j=0=j_{p h}+\sum_{k=1}^{p} j_{0, k}\left(1-\exp \left(\frac{q \times i V_{o c}}{m_{k} k_{B} T}\right)\right)-\frac{i V_{o c}}{\rho_{c, t o t}},
$$

with $\rho_{c, \text { tot }}$ the equivalent specific contact (shunt) resistance the cell is subjected to. Here we assume that, at least near opencircuit conditions, a single bulk recombination process with an ideality factor $m$ dominates so that:

$$
0=j_{p h}+j_{0}\left(1-\exp \left(\frac{q \times i V_{o c}}{m k_{B} T}\right)\right)-\frac{i V_{o c}}{\rho_{c, t o t}} \approx j_{p h}-j_{0} \times \exp \left(\frac{q \times i V_{o c}}{m k_{B} T}\right)-\frac{i V_{o c}}{\rho_{c, t o t}} .
$$

The maximal implied voltage of such a cell is achieved for $\rho_{c, t o t}=+\infty$, corresponding to perfect contact passivation, so that:

$$
\begin{gathered}
i V_{o c, \max }=\frac{m k_{B} T}{q} \ln \left(\frac{j_{p h}}{j_{0}}\right), \\
j_{p h}=j_{0} \times \exp \left(\frac{q \times i V_{o c, \text { max }}}{m k_{B} T}\right) .
\end{gathered}
$$

Considering a finite yet high enough $\rho_{c, t o t}$ to ensure $i V_{o c}=(1-\Psi) \times i V_{o c, \max }$ with $0<\Psi \ll 1(\Psi<0.001$ in our case of interest), combining Equations (B2) and (B3b) gives:

$$
j_{p h}\left(1-\exp \left(-\frac{q \Psi i V_{o c, \text { max }}}{m k_{B} T}\right)\right)=\frac{(1-\Psi) i V_{o c, \text { max }}}{\rho_{c, t o t}} .
$$

Using a Taylor series approximation of the exponential term, we then have:

$$
1-\left(1-\frac{q \Psi i V_{o c, \max }}{m k_{B} T}+O_{1}\left(\Psi^{2}\right)\right)=\frac{(1-\Psi) i V_{o c, \max }}{j_{p h} \rho_{c, t o t}}
$$

with $\left|O_{1}\left(\Psi^{2}\right)\right|$ at most equal to some constant times $\Psi^{2}$ when $\Psi$ is close enough to zero (in the present case the error term $O_{1}\left(\Psi^{2}\right)$ is positive and satisfies $\left|O_{1}\left(\Psi^{2}\right)\right|<\frac{1}{2}\left(\frac{q \Psi i V_{o c, \max }}{m k_{B} T}\right)^{2}$ for $\left.\frac{q \Psi i V_{o c, m a x}}{m k_{B} T}<1\right)$. Thus, for $\Psi$ small enough, we have:

$$
\rho_{c, t o t}=\frac{m k_{B} T}{j_{p h} q \Psi} \times \frac{1-\Psi}{1-\frac{m k_{B} T}{q \Psi i V_{o c, \max }} \times O_{1}\left(\Psi^{2}\right)}=\frac{m k_{B} T}{j_{p h} q \Psi} \times \frac{1-\Psi}{1-O_{2}(\Psi)}=\frac{m k_{B} T}{j_{p h} q \Psi}\left(1+\frac{O_{2}(\Psi)-\Psi}{1-O_{2}(\Psi)}\right)
$$


with $\left|O_{2}(\Psi)\right|$ at most equal to some constant times $\Psi$ when $\Psi$ is close enough to zero (in the present case the error term $O_{2}(\Psi)$ is positive and satisfies $\left|\mathrm{O}_{2}(\Psi)\right|<\frac{q \Psi i V_{o c, \max }}{2 m k_{B} T}$ for $\left.\frac{q \Psi i V_{o c, \max }}{m k_{B} T}<1\right)$.

The residual error term $\frac{O_{2}(\Psi)-\Psi}{1-O_{2}(\Psi)}$ converges toward zero when $\Psi$ converges towards zero and an upper limit can easily be calculated for it. In the case of an excellent crystalline silicon absorber at $300 \mathrm{~K}$ with $i V_{o c, \max }=750 \mathrm{mV}$ and bulk recombination dominated by Auger recombination with an ideality factor of $2 / 3,\left|O_{2}(\Psi)\right|<22 \Psi$ and, to achieve $\Psi=0.001$ (i.e. $i V_{o c}=0.999 \times i V_{o c, \max }>749 \mathrm{mV}$ ), we need $\rho_{c, t o t}=1000 m k_{B} T / j_{p h} q$ with an error of less than $2.2 \%$.

\section{REFERENCES}

$1 \quad$ P. Würfel and U. Würfel, Physics of solar cells : from basic principles to advanced concepts, 2nd, updated and expanded ed. (Wiley-VCH, Weinheim, 2009).

O. D. Miller, E. Yablonovitch, and S. R. Kurtz, IEEE Journal of Photovoltaics 2, 303 (2012).

R. T. Ross, The Journal of Chemical Physics 46, 4590 (1967).

A. G. Aberle, Progress in Photovoltaics 8, 473 (2000).

A. Cuevas and D. Yan, IEEE Journal of Photovoltaics 3, 916 (2013).

U. Würfel, A. Cuevas, and P. Würfel, IEEE Journal of Photovoltaics 5, 461 (2015).

Z. C. Holman, P. L. Koswatta, and M. Boccard, in SiliconPV 2015: $5^{\text {th }}$ International Conference on Silicon Photovoltaics, Konstanz, Germany, 2015.

P. L. Koswatta, M. Boccard, and Z. C. Holman, in IEEE $42^{\text {nd }}$ Photovoltaic Specialists

Conference, New Orleans, LA, USA, 2015, p. 2431.

P. L. Koswatta, Master Thesis, Arizona State University, 2016.

S. Glunz, M. Bivour, C. Messmer, F. Feldmann, R. Müller, C. Reichel, A. Richter, F. Schindler, J. Benick, and M. Hermle, in IEEE $44^{\text {th }}$ Photovoltaic Specialists Conference, Washington, D.C., USA, 2017.

A. Cuevas, Energy Procedia 55, 53 (2014).

D. E. Kane and R. M. Swanson, in IEEE $18^{\text {th }}$ Photovoltaic Specialists Conference, Las Vegas, NV, USA, 1985, p. 578.

D. L. Meier and D. K. Schroder, IEEE Transactions on Electron Devices 31, 647 (1984).

A. Fell, F. Feldmann, C. Messmer, M. Bivour, M. C. Schubert, and S. W. Glunz, IEEE Journal of Photovoltaics 8, 1546 (2018).

J. Bullock, PhD Thesis, Australia National University, 2016.

R. Brendel and R. Peibst, IEEE Journal of Photovoltaics 6, 1413 (2016).

E. T. Roe, K. E. Egelhofer, and M. C. Lonergan, ACS Applied Energy Materials 1, 1037 (2018).

E. T. Roe, K. E. Egelhofer, and M. C. Lonergan, Journal of Applied Physics 125 (2019).

A. Spies, M. List, T. Sarkar, and U. Würfel, Advanced Energy Materials 7 (2017).

W. Tress, K. Leo, and M. Riede, Physical Review B 85 (2012).

C. Messmer, M. Bivour, J. Schon, S. W. Glunz, and M. Hermle, IEEE Journal of Photovoltaics 8, 456 (2018). 
H. Haug, B. R. Olaisen, Ø. Nordseth, and E. S. Marstein, Energy Procedia 38, 72 (2013). R. Brendel and C. Kruse, in $28^{\text {th }}$ Workshop on Crystalline Silicon Solar Cells \& Modules: Materials and Processes, Winter Park, CO, USA, 2018, p. 58.

A. Cuevas, Y. Wan, D. Yan, C. Samundsett, T. Allen, X. Zhang, J. Cui, and J. Bullock, Solar Energy Materials and Solar Cells 184, 38 (2018).

A. Onno, C. Chen, and Z. C. Holman, in IEEE $46^{\text {th }}$ Photovoltaic Specialists Conference, Chicago, IL, USA, 2019.

F. Feldmann, M. Bivour, C. Reichel, M. Hermle, and S. W. Glunz, Solar Energy Materials and Solar Cells 120, 270 (2014).

B. Hallam, B. Tjahjono, T. Trupke, and S. Wenham, Journal of Applied Physics 115, 044901 (2014).

A. Augusto, S. Y. Herasimenka, R. R. King, S. G. Bowden, and C. Honsberg, Journal of Applied Physics 121, 205704 (2017).

D. Lachenal, D. Baetzner, W. Frammelsberger, B. Legradic, J. Meixenberger, P. Papet, B. Strahm, and G. Wahli, Energy Procedia 92, 932 (2016).

J. Schmidt, R. Peibst, and R. Brendel, Solar Energy Materials and Solar Cells 187, 39 (2018).

A. Descoeudres, Z. C. Holman, L. Barraud, S. Morel, S. De Wolf, and C. Ballif, IEEE Journal of Photovoltaics 3, 83 (2013).

C. Deibel, A. Wagenpfahl, and V. Dyakonov, Physica Status Solidi-Rapid Research Letters 2, 175 (2008).

T. Kirchartz, B. E. Pieters, K. Taretto, and U. Rau, Physical Review B 80, 035334 (2009).

T. Kirchartz and U. Rau, Advanced Energy Materials 8, 1703385 (2018).

B. Demaurex, S. De Wolf, A. Descoeudres, Z. C. Holman, and C. Ballif, Applied Physics Letters 101, 171604 (2012).

A. Cuevas, T. Allen, J. Bullock, W. Yimao, Y. Di, and Z. Xinyu, in IEEE $42^{\text {nd }}$ Photovoltaic Specialist Conference, 2015. 
Left electrode
Left contact

(hole)

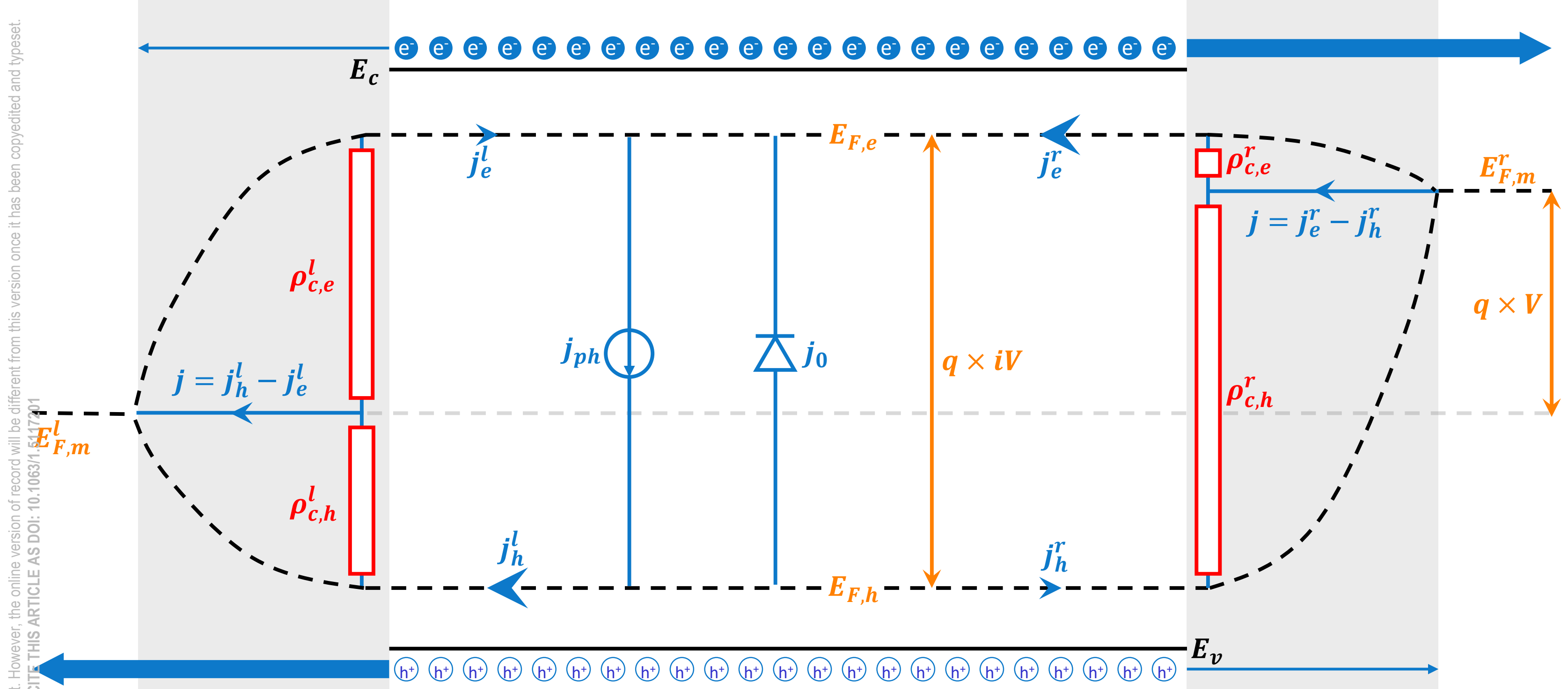



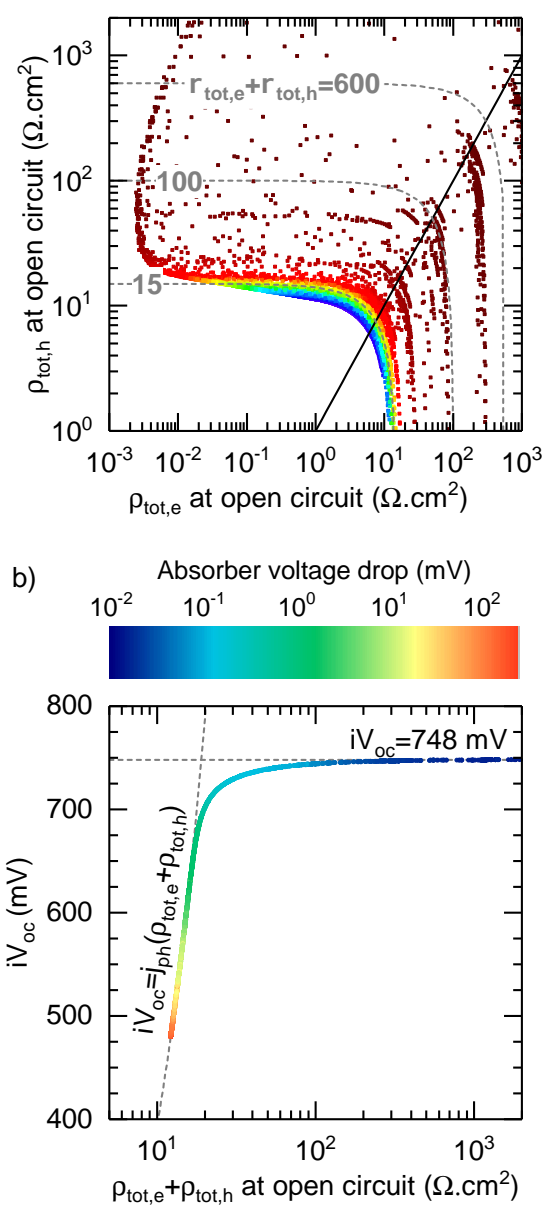


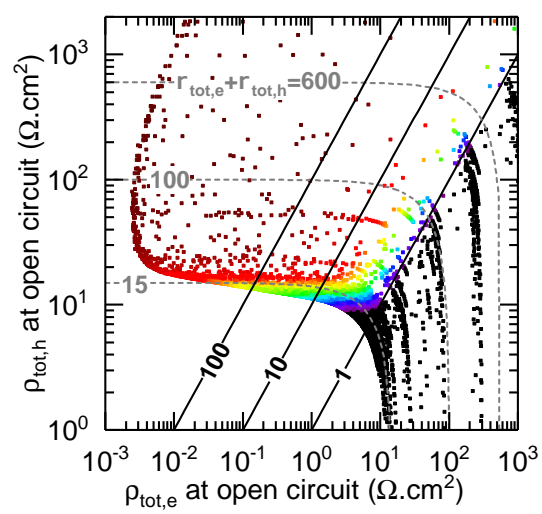

b)

$\mathrm{iV}_{\text {oc }}(\mathrm{mV})$

$\begin{array}{lllllll}450 & 500 & 550 & 600 & 650 & 700 & 750\end{array}$

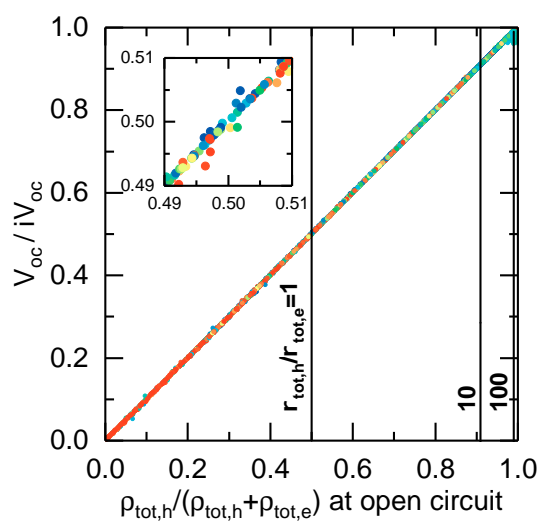


a)
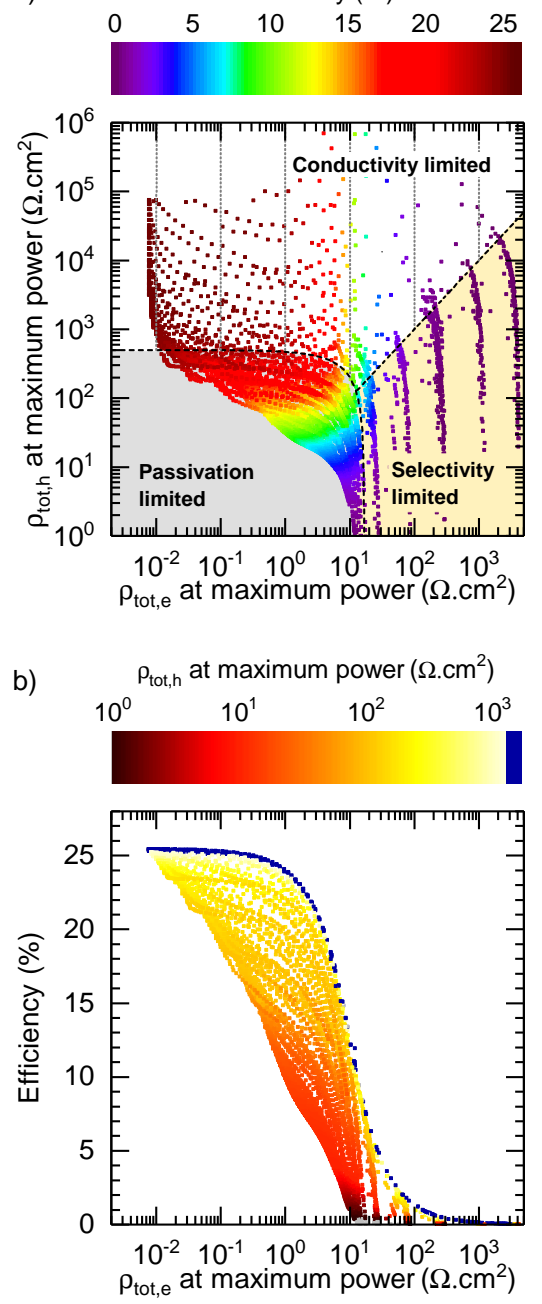

C) $\rho_{\text {tot, } \mathrm{e}} /\left(\rho_{\text {tot, } \mathrm{e}}+\rho_{\text {tot, }, \mathrm{h}}\right)$ at maximum power $\left(\Omega . \mathrm{cm}^{2}\right)$

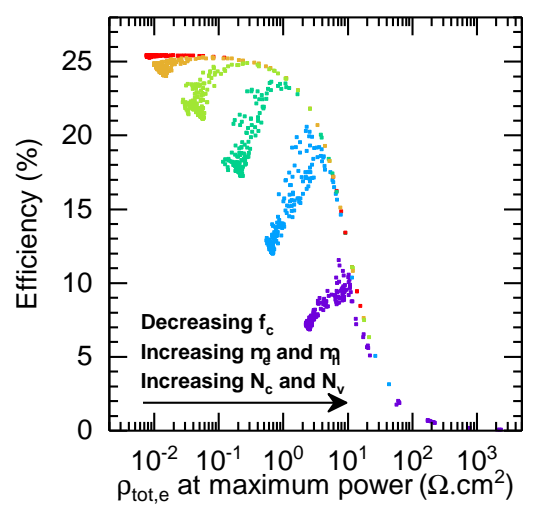

